\title{
Unmasking Silent Endothelial Activation in the Cardio- vascular System Using Molecular Magnetic Resonance Imaging
}

\author{
Julie Belliere ${ }^{1,2}$, Sara Martinez de Lizarrondo ${ }^{3}$, Robin P. Choudhury ${ }^{4}$, Aurélien Quenault ${ }^{3}$, Audrey Le \\ Béhot $^{3}$, Christine Delage 2,5, Dominique Chauveau ${ }^{1,2,6}$, Joost P. Schanstra1,2, Jean-Loup Bascands ${ }^{1,2}$, Denis \\ Vivien $^{3}$, Maxime Gauberti ${ }^{3 凶}$ \\ 1. Inserm, Institut National de la Santé et de la Recherche Médicale (INSERM), INSERM U1048, Institute of Cardiovascular and Metabolic Diseases, \\ Toulouse, France. \\ 2. Université Paul Sabatier, Toulouse, France. \\ 3. Institut National de la Santé et de la Recherche Médicale (INSERM), INSERM UMR-S U919, Serine Proteases and Pathophysiology of the Neu- \\ rovascular Unit, Caen, France. \\ 4. Division of Cardiovascular Medicine Radcliffe, Department of Medicine, University of Oxford, Oxford, UK. \\ 5. Department of Microsurgery, Institut National de la Santé et de la Recherche Médicale (INSERM UMS006), Toulouse, France. \\ 6. Department of Nephrology and Organ Transplantation, Faculté Purpan, CHU Rangueil, Toulouse, France
}

$\triangle$ Corresponding author: Maxime Gauberti, PhD., Serine Proteases and Pathophysiology of the Neurovascular Unit INSERM U919, GIP Cyceron / Université de Caen, Bd Henri Becquerel, 14074 Caen, France. Phone: 00332314701 66; Fax: 00332314702 22; Email: gauberti@cyceron.fr

(c) 2015 Ivyspring International Publisher. Reproduction is permitted for personal, noncommercial use, provided that the article is in whole, unmodified, and properly cited. See http://ivyspring.com/terms for terms and conditions.

Received: 2015.02.09; Accepted: 2015.07.15; Published: 2015.08.08

\begin{abstract}
Endothelial activation is a hallmark of cardiovascular diseases, acting either as a cause or a consequence of organ injury. To date, we lack suitable methods to measure endothelial activation in vivo. In the present study, we developed a magnetic resonance imaging (MRI) method allowing non-invasive endothelial activation mapping in the vasculature of the main organs affected during cardiovascular diseases. In clinically relevant contexts in mice (including systemic inflammation, acute and chronic kidney diseases, diabetes mellitus and normal aging), we provided evidence that this method allows detecting endothelial activation before any clinical manifestation of organ failure in the brain, kidney and heart with an exceptional sensitivity. In particular, we demonstrated that diabetes mellitus induces chronic endothelial cells activation in the kidney and heart. Moreover, aged mice presented activated endothelial cells in the kidneys and the cerebrovasculature. Interestingly, depending on the underlying condition, the temporospatial patterns of endothelial activation in the vascular beds of the cardiovascular system were different. These results demonstrate the feasibility of detecting silent endothelial activation occurring in conditions associated with high cardiovascular risk using molecular MRI.
\end{abstract}

Key words: Inflammation, MPIO, USPIO, VCAM-1, ICAM-1.

\section{Introduction}

Endothelial activation is a stereotyped response of the macro- and microvasculature to a stress or an injury. It is associated with most forms of cardiovascular diseases, such as diabetes, coronary artery disease, chronic renal failure and stroke[1]. Endothelial activation involves several phenotypic changes of the luminal surface of endothelial cells that are necessary for efficient leukocytes adherence and diapedesis to injured tissues. Interestingly, mice with blunted endothelial activation achieved by Nuclear Factor- $\mathrm{BB}$ blockade present an increased lifespan and are resistant to cardiovascular diseases[2, 3]. Thus, endo- 
thelial activation represents a key therapeutic target for preventing and treating cardiovascular diseases. However, current diagnostic approaches for its clinical and preclinical evaluation remain limited.

Most clinical studies have estimated the mean systemic level of endothelial activation by looking at soluble biomarkers such as E-selectin, intracellular adhesion molecule-1 (ICAM-1) or vascular cell adhesion molecule-1 (VCAM-1). Measurement of soluble VCAM-1 is especially useful to reveal endothelial activation, since it is not expressed by quiescent endothelial cells and is steadily and strongly expressed after activation[4]. However, these methods are limited, since the vascular beds of every target organ may be differentially affected. Moreover, many other mechanisms may increase plasmatic level of endothelial activation biomarkers, such as kidney insufficiency or nycthemeral fluctuations.

To overcome these limitations, an ideal diagnostic procedure should allow in situ, fast, sensitive, specific and multi-organ detection of endothelial activation biomarkers[5, 6]. MRI is the method of choice for high-resolution imaging of deep tissues, without ionizing radiation. However, classical contrast agents such as gadolinium chelate or ultrasmall superparamagnetic particles of iron oxide (USPIO) are prone to unspecific extravasation because of their small sizes. Moreover, because of their lower relaxivity, they induce only limited signal changes on MRI and require high local concentrations to be detectable. Therefore, their characteristics in terms of sensitivity and specificity are not ideal for fast and multi-organ imaging[7-9]. Larger particles (micron-sized particles of iron oxide, MPIO) have provided better specificity since their larger size prevents unspecific extravasation[1]. Unfortunately, their sensitivity to reveal proteins or cells on the activated endothelial surface remains low, even in conditions associated with a strong (and probably clinically irrelevant) inflammatory response[1, 7]. Moreover, in vivo imaging of the heart remains challenging and, to date, we have limited methods available for cardiac molecular MRI[10].

In the present study, we developed an imaging strategy to achieve highly sensitive mapping of endothelial activation using a formulation of MPIO targeted against VCAM-1 (MPIO- $\alpha$ VCAM-1)[11]. We first characterized the pharmacokinetic of the binding of MPIO- $\alpha$ VCAM-1 in vivo in the different vascular beds using fast $\mathrm{T}^{*}$-weighted imaging. Then, we performed high-resolution MRI to map endothelial activation in the vasculature of target organs (heart, brain and kidneys) in experimental models of clinical conditions where acute or chronic endothelial activation were suspected to occur, including systemic inflammation, diabetes mellitus, acute kidney injury (AKI), chronic kidney disease and normal aging.

\section{Methods}

\section{Animals}

Rhabdomyolysis and diabetes studies were performed on 8-week-old C57B16J male mice (Janvier, Le Genest-Saint-Isle, France). Systemic inflammation (Lipopolysaccharide, LPS) and Subtotal nephrectomy studies were performed on 8-week-old male Swiss mice (Janvier, France). Animals were maintained under specific pathogen-free conditions at the Centre Universitaire de Ressources Biologiques (CURB, Basse-Normandie, France) and all had free access to food and tap water before and after the procedures. Aged animals were male swiss mice (18 month-old) maintained under the same conditions at the CURB. Experiments were approved by the local ethical committee (C-31.555-07, US006 CREFRE (CEEA-122)) and were performed in accordance with the French (Decree 87/848) and the European Communities Council (Directive 86/609) guidelines.

\section{LPS-induced systemic inflammation model}

Swiss mice received an intra-peritoneal (i.p.) injection of LPS from E. coli (0.1 to $1 \mathrm{mg} / \mathrm{kg}$, 0111:B4, Sigma-Aldrich, Lisle d'Abeau, France). MRI was performed 48 hours after LPS administration.

\section{Rhabdomyolysis-induced acute kidney injury (AKI)}

To induce rhabdomyolysis, the animals were injected intramuscularly, on each thigh caudal muscle, with $7.5 \mathrm{~mL} / \mathrm{kg} 50 \%$ Glycerol $(99,5 \% \mathrm{~m} / \mathrm{v}$, VWR International S.A.S., France) or saline as a control[12, 13].

\section{Subtotal (5/6) nephrectomy (SNX)}

Mice were subjected to SNX as previously described[14], with minor modifications. The procedure was performed in anesthetized mice (ketamine (100 $\mathrm{mg} / \mathrm{kg})$, xylazine $(10 \mathrm{mg} / \mathrm{kg})$, buprenorphine (100 $\mu \mathrm{g} / \mathrm{kg}$ ) i.p.). The left kidney was exposed via a flank incision and both poles were excised (approximately two thirds of the kidney), leaving a small amount of renal tissue around the left ureter and hilar vessels. Blood loss was minimized by the application of gelatin sponges. Finally, the right kidney was removed, leaving $20-25 \%$ of the total renal mass.

\section{Streptozotocin-induced diabetes model}

Mice received i.p. injections of streptozotocin (STZ $50 \mathrm{mg} / \mathrm{kg}$, sigma) during 5 days after 4 hours of fasting. Mice have been tested for sufficient levels of hyperglycemia $(>300 \mathrm{mg} / \mathrm{dl})$ two days after injection and 4 weeks post-injection. 


\section{Targeting-moiety conjugation to MPIO}

Microparticles of iron oxide (MPIO; diameter $1.08 \mu \mathrm{m})$ with p-toluenesulphonyl reactive surface groups (Invitrogen) were covalently conjugated to purified monoclonal rat anti-mouse monoclonal antibodies for VCAM-1. Briefly, purified monoclonal rat anti-mouse antibodies for VCAM-1 (clone A(429); BD-Bioscience) or control rat IgG (Jackson ImmunoResearch) were covalently conjugated to MPIOs in borate buffer ( $\mathrm{pH} 9.5$ ), by incubation at $37^{\circ} \mathrm{C}$ for $48 \mathrm{~h}$. $40 \mu \mathrm{g}$ of targeting molecule was used for the coating of $1 \mathrm{mg}$ of reactive MPIOs. MPIOs were then washed in phosphate buffered saline (PBS) containing 0.5\% bovine serum albumin (BSA) at $4{ }^{\circ} \mathrm{C}$ and incubated for $24 \mathrm{~h}$ at room temperature, to block the remaining active groups. MPIOs were rinsed in PBS (0.1\% BSA). To disperse MPIO aggregates, a sonication procedure at low intensity was performed one time, immediately after antibody labelling, for 60 seconds. Thereafter, MPIO were stored at $4^{\circ} \mathrm{C}$ in a PBS buffer under constant agitation to prevent settling and aggregate formation. We used each MPIO batches for approximately 1 month after antibody labeling and, without further sonication, no aggregate were visible on MRI acquisition (Fig. S1). Finally, monodisperse MPIOs (as assessed by epifluorescence microscopy) were stored at $4^{\circ} \mathrm{C}$. Using a similar protocol of antibody conjugation, Jefferson et al. measured that the density of targeting antibodies on the surface of MPIO was 27,100 \pm 1920 molecules per MPIO, equivalent to 8500 molecules $\mu \mathrm{m}^{-2}$.[15]

Mice received intravenous injection of $1.0 \mathrm{mg} / \mathrm{kg}$ (equivalent $\mathrm{Fe}$ ) of conjugated MPIOs for contrast-enhanced MRI. Imaging was performed 10 minutes after MPIO administration. This delay between injection and imaging was chosen on the basis of pharmacokinetic studies showing that MPIO signal from the vasculature is short-lived and that MPIO-binding occurs during the first pass of the contrast agent across kidney, heart and brain vessels (see results section). $\mathrm{R} 1$ and $\mathrm{R} 2^{*}$ relaxivities of the $1 \mu \mathrm{m}$ MPIO used in the present study were $16.6 \mathrm{~s}^{-1} \mathrm{mM}^{-1}$ and $1123 \mathrm{~s}^{-1} \mathrm{mM}^{-1}$, respectively, as measured in $2 \%$ agarose gels at $7 \mathrm{~T}$.

\section{Magnetic Resonance Imaging (MRI)}

MRI Experiments were carried out on a Pharmascan $7 \mathrm{~T} / 12 \mathrm{~cm}$ system using surface coils (Bruker, Germany). T2-weighted images were acquired using a multi-slice multi-echo (MSME) sequence: effective TE/TR $51 \mathrm{~ms} / 2500 \mathrm{~ms}$ with $70 \mu \mathrm{m}^{*} 70 \mu \mathrm{m} * 500 \mu \mathrm{m}$ spatial resolution, 8 acquired echos with 1 computed image.[16, 17] High resolution imaging: 3D respiration-gated $\mathrm{T} 2{ }^{*}$-weighted gradient echo imaging with flow compensation (GEFC, spatial resolution of
$93 \mu \mathrm{m} * 70 \mu \mathrm{m} * 70 \mu \mathrm{m}$ interpolated to an isotropic resolution of $70 \mu \mathrm{m}$ ) with TE/TR $12.6 \mathrm{~ms} / 200 \mathrm{~ms}$ and a flip angle (FA) of $24^{\circ}$ was performed to visualize MPIOs (acquisition time $=8 \mathrm{~min}$ ). Cardiac imaging was performed using both respiratory and electrocardiographic gatings. All high-resolution $\mathrm{T} 2{ }^{*}$-weighted images presented in this study are minimum intensity projections of 4 consecutive slices (yielding a $\mathrm{Z}$ resolution of $280 \mu \mathrm{m}$ ). Fast imaging was performed using a T2* weighted Fast low angle shot sequence with TR/TE 25ms/5.7ms and matrix size 192x160 (yielding a temporal resolution of $4.0 \mathrm{~s}$ using a k-space acceleration factor of 2), Field of view $=19.2 \times 16.0 \mathrm{~mm}, 100$ time-points. When appropriate, mice randomly received MPIO-IgG or MPIO- $\alpha$ VCAM-1. Although MPIO injections were performed outside the magnet, the positions of the mice during pre-MPIO and post-MPIO acquisitions did not change (the mice were placed exactly at the same position and were not removed from the animal holder).

\section{MRI Analysis}

The first step was to crop the initial MRI in a 230x70 pixels picture, including either both kidneys, the brain or the heart. Then, the organs were manually segmented in imageJ. Lastly, 3D Otsu automated threshold is applied using ImageJ software and the signal void volume is computed (in percentage of the total organ volume). Results are presented as the volume of MPIOs-induced signal void divided by the volume of the structure of interest (in percentage). This procedure is presented for the kidneys in Fig. S2. All analyses were performed using ImageJ v1.49e.

\section{Competition experiments}

When appropriate, mice received $2.5 \mathrm{mg} / \mathrm{kg}$ of control isotype IgG (Jackson Immunoresearch, USA) or anti-VCAM-1 antibody (clone A(429), BD Bioscience) 30 minutes before MPIO- $\alpha$ VCAM-1 injection to block the MPIO-binding sites. MRI was thereafter performed as previously described.

\section{Intra-striatal LPS injection}

Swiss mice received an intra-striatal injection of LPS from E. coli (1.5 $\mu \mathrm{g} /$ mouse, 0111:B4, Sigma-Aldrich, Lisle d'Abeau, France) that was performed after placing Swiss male mice in a stereotaxic frame (coordinates: $0.5 \mathrm{~mm}$ anterior, $2.0 \mathrm{~mm}$ lateral, -3 $\mathrm{mm}$ ventral to the bregma). Solutions were injected by the use of a glass micropipette. MRI was performed 24 hours after LPS administration as previously described.

\section{Immunohistochemistry}

Immunohistochemistry was performed as previously described[11, 18, 19]. Deeply anesthetized 
mice were transcardially perfused with cold heparinized saline $(15 \mathrm{~mL})$ followed by $150 \mathrm{~mL}$ of fixative (PBS 0.1 M. pH 7.4 containing 2\% paraformaldehyde and $0.2 \%$ picric acid). Brains were post-fixed (18 hours; $4^{\circ} \mathrm{C}$ ) and cryoprotected (sucrose $20 \%$ in veronal buffer; 24 hours; $4^{\circ} \mathrm{C}$ ) before freezing in Tissue-Tek (Miles Scientific, Naperville, IL, USA). Cryomicrotome-cut transversal sections $(8-10 \mu \mathrm{m})$ were collected on poly-lysine slides and stored at $-80^{\circ} \mathrm{C}$ before processing. Sections were co-incubated overnight with rat monoclonal anti-mouse VCAM-1 (1:1000; from BD Biosciences), goat anti-collagen-type IV (1:1500; Southern Biotech), chicken anti-MAP-2 (1:8000; Abcam), goat anti-Iba-1 (1:800; Abcam) or rabbit anti-GFAP (1:800; Dako) in veronal buffer ( $\mathrm{pH} 7.4$ ). Primary antibodies were revealed using Fab'2 fragments of Donkey anti-rat, goat, chicken or mouse IgG linked to FITC, TRITC or DyLight 629 (1:500, Jackson ImmunoResearch, West Grove, USA). Washed sections were coverslipped with antifade medium containing DAPI and images were digitally captured using a Leica DM6000 microscope-coupled coolsnap camera and visualized with Metavue 5.0 software (Molecular Devices, USA) and further processed using Image 1.49 e software $(\mathrm{NIH})$. A vessel was considered VCAM-1 positive when the fluorescence signal was distinguishable from the background (parenchymal auto-fluorescence). All vessels (micro and macro-sized) were included in the analyses, except for the heart where only large vessels were considered since the auto-fluorescence from the cardiac tissue prevented reliable analysis of microvessel fluorescence. All analyses were performed at 200x magnification. All analyses were performed blinded to the experimental data.

\section{Statistical analysis}

Results are presented as the mean \pm SD. Statistical analyses were performed using Kruskal-Wallis (for multiple comparisons) followed by Mann-Whitney's U-test. When comparing two groups, a p-value $<0.05$ was considered to be significant.

\section{Results}

\section{Pharmacokinetic characterization of MPIO- $\alpha$ VCAM- 1 binding to activated endo- thelium in vivo}

To study the pharmacokinetic of MPIO- $\alpha$ VCAM-1 binding to activated endothelium, we first induced a systemic inflammatory response in mice by intraperitoneal injection of LPS $(1 \mathrm{mg} / \mathrm{kg})$. This led to massive endothelial activation in the kid- neys, the brain and the heart 48 hours after injection, as revealed by VCAM-1 overexpression on immunohistofluorescence (Fig. S3). Then we injected MPIO- $\alpha$ VCAM-1 in LPS-treated mice during continuous fast $\mathrm{T} 2{ }^{*}$-weighted imaging to observe MPIO- $\alpha$ VCAM-1 biodistribution, first in the kidney and the inferior vena cava, then in the brain, to confirm our findings in different organs. We performed low spatial resolution $\mathrm{T} 2^{*}$-weighted imaging to reach a high temporal resolution (4 seconds), which was necessary to reliably image the fast distribution of MPIO- $\alpha$ VCAM-1. As shown on Fig. S4, MPIO- $\alpha$ VCAM-1 administration induced transient vascular hyposignal in the inferior vena cava and renal vessels. Quantitative analyses revealed that MPIO- $\alpha$ VCAM-1-induced signal void in the blood is short-lived, with complete recovery of the vascular signal after less than 60 seconds, confirming previous studies.[20] These data provide evidence that MPIO- $\alpha$ VCAM-1 are completely cleared from the plasma in less than 60 seconds, suggesting a very short plasmatic half-life. Standard measurements of plasmatic half-life may allow to further refine these parameters.

In the kidney parenchyma, the maximal decrease in signal intensity induced by MPIO- $\alpha$ VCAM- 1 was reached in $\sim 12$ seconds and remained stable thereafter. Thus, most MPIO- $\alpha$ VCAM- 1 bind to the activated endothelium of the kidney of LPS-treated mice during their first passage through the organ, demonstrating a first-pass effect of the contrast agent on signal intensity. We confirmed these results in the brain, where the same first-pass effect was observed (Fig. S5). Unbound particles are rapidly cleared by the liver and the spleen from the vasculature (Fig. S6). Altogether, these results demonstrated that most MPIO- $\alpha$ VCAM-1 bind to activated endothelium during their first pass through the vasculature, that they remain firmly attached for several minutes after their binding on activated endothelium and that they are rapidly cleared from the blood by the liver and the spleen (reticulo-endothelial system). These pharmacokinetic characteristics allow, therefore, to perform imaging shortly after MPIO- $\alpha$ VCAM- 1 administration and with a large time-window before MPIO- $\alpha$ VCAM-1 detachment from the vascular wall.

\section{Mapping endothelial cell activation in a model of systemic inflammation}

Then, we evaluated the sensitivity and specificity of MPIO- $\alpha$ VCAM-1 to reveal endothelial cell activation using the same model of systemic inflammation in mice by intraperitoneal injection of LPS (1 $\mathrm{mg} / \mathrm{kg}$ ). In contrast to the previous experiments, in which the sensitivity of the fast $\mathrm{T} 2{ }^{*}$-weighted imaging 
was relatively low, we performed high-resolution $\mathrm{T} 2{ }^{*}$-weighted imaging to reach higher level of sensitivity. We successively performed kidney, brain and heart imaging in the same animals (acquisition times of 8,12 and 10 minutes respectively), with and without MPIO- $\alpha$ VCAM-1 administration at 48 hours post-LPS (Fig. 1A). Imaging was performed 10 minutes after MPIO administration (delay required to secure the mouse inside the magnet and perform initial MRI adjustments), allowing complete clearance of the circulating unbound MPIO according to our pharmacokinetic data. For each vascular bed, we evaluated sensitivity and specificity of the method using untargeted MPIOs (MPIO-IgG), dose-response experiments and immunohistological confirmation of the results.

Starting with the kidneys, as shown in Fig. 1, pre-contrast conventional $\mathrm{T} 2$ - and $\mathrm{T} 2{ }^{*}$-weighted imaging failed to reveal any alteration in LPS-treated mice. Therefore, conventional imaging was unable to discriminate LPS-treated from control mice. In contrast, following MPIO- $\alpha$ VCAM-1 administration, signals on $\mathrm{T} 2{ }^{*}$-weighted images dramatically dropped only in LPS-treated mice (Fig. 1B-C). MPIO-induced signal changes manifested as signal void in both cortical and medullar regions of the kidneys (Fig. 1C). This was confirmed by immunohistological analyses of the kidneys (removed immediately after MRI acquisition), revealing numerous MPIO- $\alpha$ VCAM- 1 in the lumen of VCAM-1-expressing kidney vessels, without any evidence of extravasated particles (Fig. 1D). To evaluate the specificity of the observed contrast-changes, we performed the same experiments in LPS-treated mice using control untargeted-MPIOs (MPIO-IgG). As shown on Fig. 1E, untargeted MPIO (MPIO-IgG) did not induce changes on $\mathrm{T} 2{ }^{*}$-weighted images. To further confirm the specificity of MPIO- $\alpha$ VCAM-1 induced signal voids, we performed competitive experiments by pre-treating the mice with anti-VCAM-1 antibodies 30 minutes before MPIO- $\alpha$ VCAM- 1 administration. This paradigm allowed blocking of the MPIO- $\alpha$ VCAM-1 binding sites on the inflamed endothelium. As shown on Fig. 1F, anti-VCAM-1 blockade dramatically reduced MPIO- $\alpha$ VCAM-1 induced signal void in the kidneys. Altogether, these three MRI experiments demonstrated the specificity of MPIO- $\alpha$ VCAM- 1 to reveal endothelial activation in the kidney.

Subsequently, to evaluate the sensitivity of the present method to unmask endothelial activation in the kidney vasculature, we performed MPIO- $\alpha$ VCAM-1 enhanced MRI after administration of different LPS dosages (from 0.1 to $0.8 \mathrm{mg} / \mathrm{kg}$ ) to mimic different grades of sepsis severity. As shown on Fig. 2A-C, contrast-enhanced imaging revealed kidney inflammation from the lowest tested dose of LPS $(0.1 \mathrm{mg} / \mathrm{kg})$. Interestingly, the volume of MPIO- $\alpha$ VCAM-1-induced signal void paralleled the amount of LPS injected, revealing a dose-response relationship. Hence, more severe are the systemic inflammation and thus the level of endothelial activation, larger are the MPIO- $\alpha$ VCAM-1-induced signal voids. Another essential point for imaging specificity is the kinetic of contrast clearance after endothelial binding. Indeed, to allow longitudinal imaging, clearance of MPIO- $\alpha$ VCAM-1 should be short enough to avoid contrast persistence between two consecutive imaging sessions. Thus, we studied the kinetic of contrast disappearance after MPIO- $\alpha$ VCAM-1 injection in LPS-treated mice $(1 \mathrm{mg} / \mathrm{kg})$. Importantly, we observed that the contrast agent was progressively cleared from the kidneys in a time-dependent manner, resulting in minimal background contrast 24 hours after MPIO- $\alpha$ VCAM-1 injection (Fig. 2D-F). This kinetic profile of contrast agent clearance allows repetitive assessment of endothelial activation in the kidneys with a minimal temporal resolution of 24 hours. Altogether, these results demonstrated the feasibility of sensitive and semi-quantitative imaging of VCAM-1 in the kidney after intravenous injection of MPIO- $\alpha$ VCAM-1.

Concerning the heart, we performed MPIO- $\alpha$ VCAM- 1 enhanced MRI using a cardiac-triggered $\mathrm{T} 2{ }^{*}$-weighted sequence. As shown on Fig. 3A, MPIO- $\alpha$ VCAM-1 enhanced MRI revealed strong VCAM-1 expression in the whole heart 48 hours after LPS administration, in line with the histological findings (Fig. S3B). To evaluate the specificity of this method, we performed the same experiments using control untargeted MPIO (MPIO-IgG). The specificity of MPIO- $\alpha$ VCAM-1 MRI was confirmed by the lack of signal void after injection of MPIO-IgG in LPS-treated mice (Fig. 3B). Moreover, as previously demonstrated for the kidneys, there was a dose-response relationship between the severity of the systemic inflammatory insult (amount of LPS administered, from $0.1-0.8 \mathrm{mg} / \mathrm{kg}$ ) and the volume of MPIO- $\alpha$ VCAM-1-induced signal void in the heart (Fig. 3C). Histological analyses confirmed the presence of MPIO- $\alpha$ VCAM- 1 in the inflamed vascular beds of the heart (including cardiac endothelium and endocardium) similarly to the results obtained in the kidneys (Fig. 3D). 
A

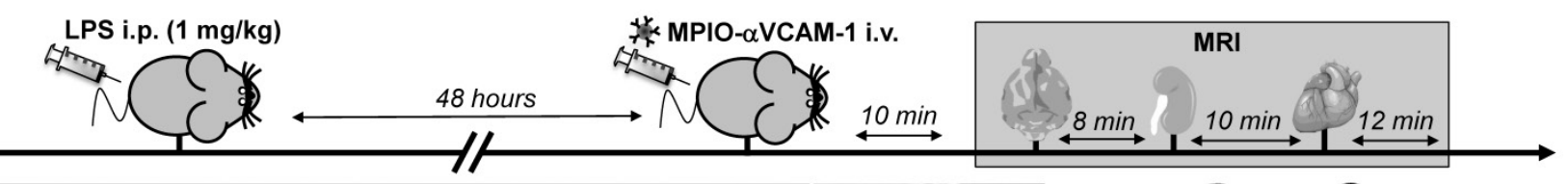

B
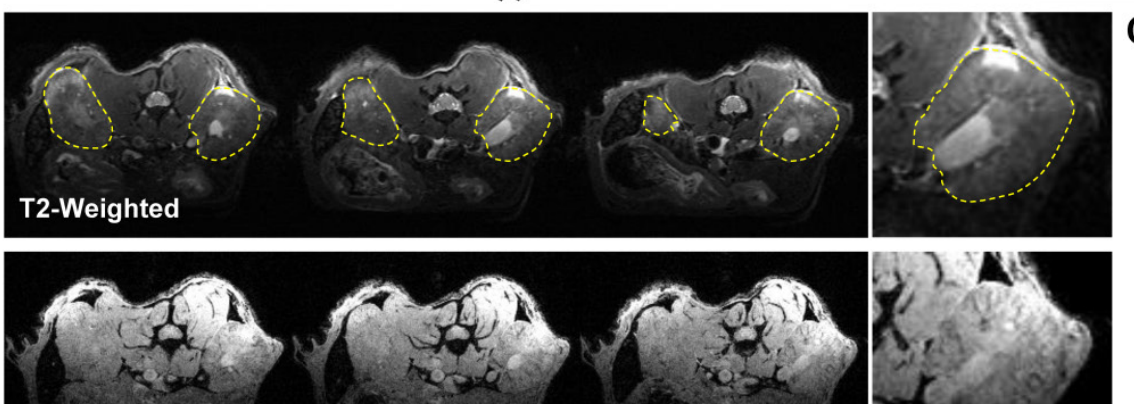

T2*-Weighted - Before MPIO- $\alpha$ VCAM-1

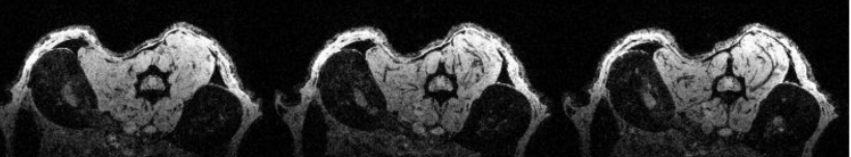

T2*-Weighted - After MPIO- $\alpha$ VCAM-1

D
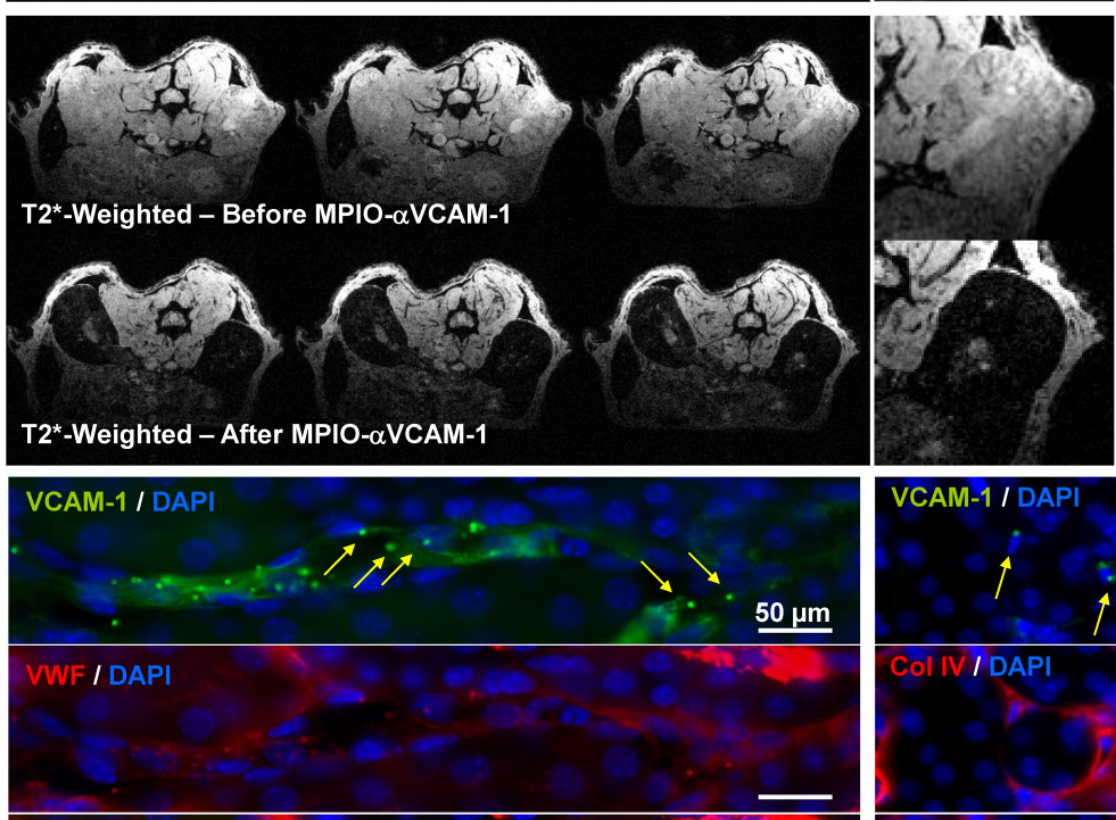

C

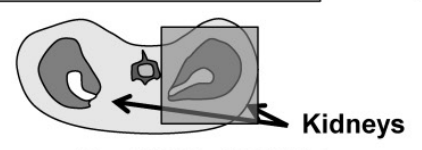

After MPIO- $\alpha$ VCAM-1
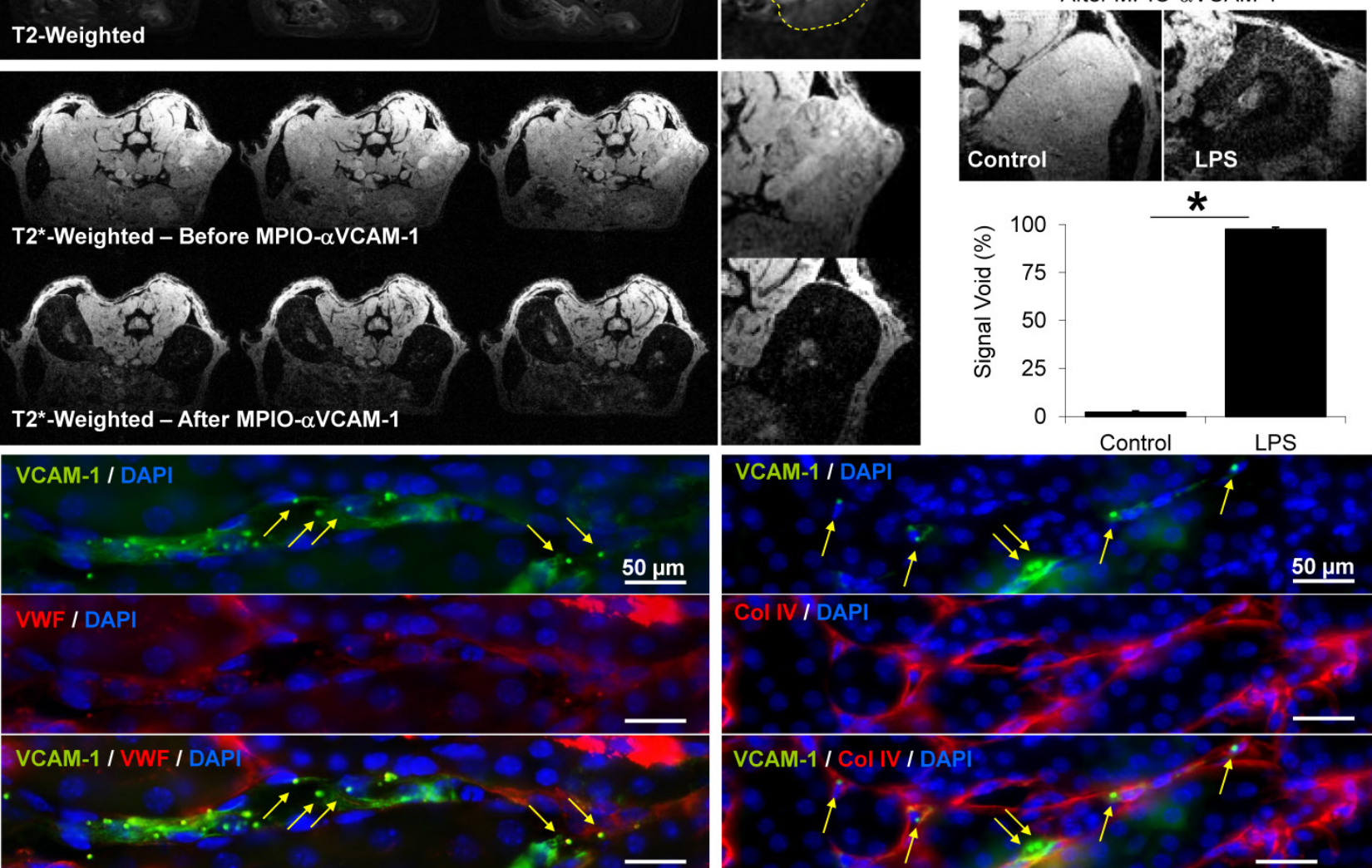

E

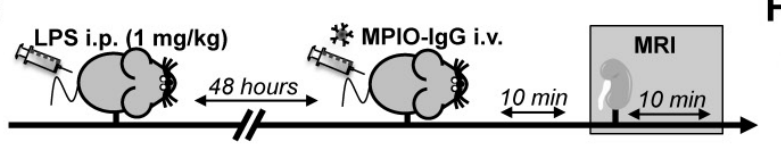

F LPS i.p. $(1 \mathrm{mg} / \mathrm{kg}) \quad \operatorname{lgG} /$ anti-
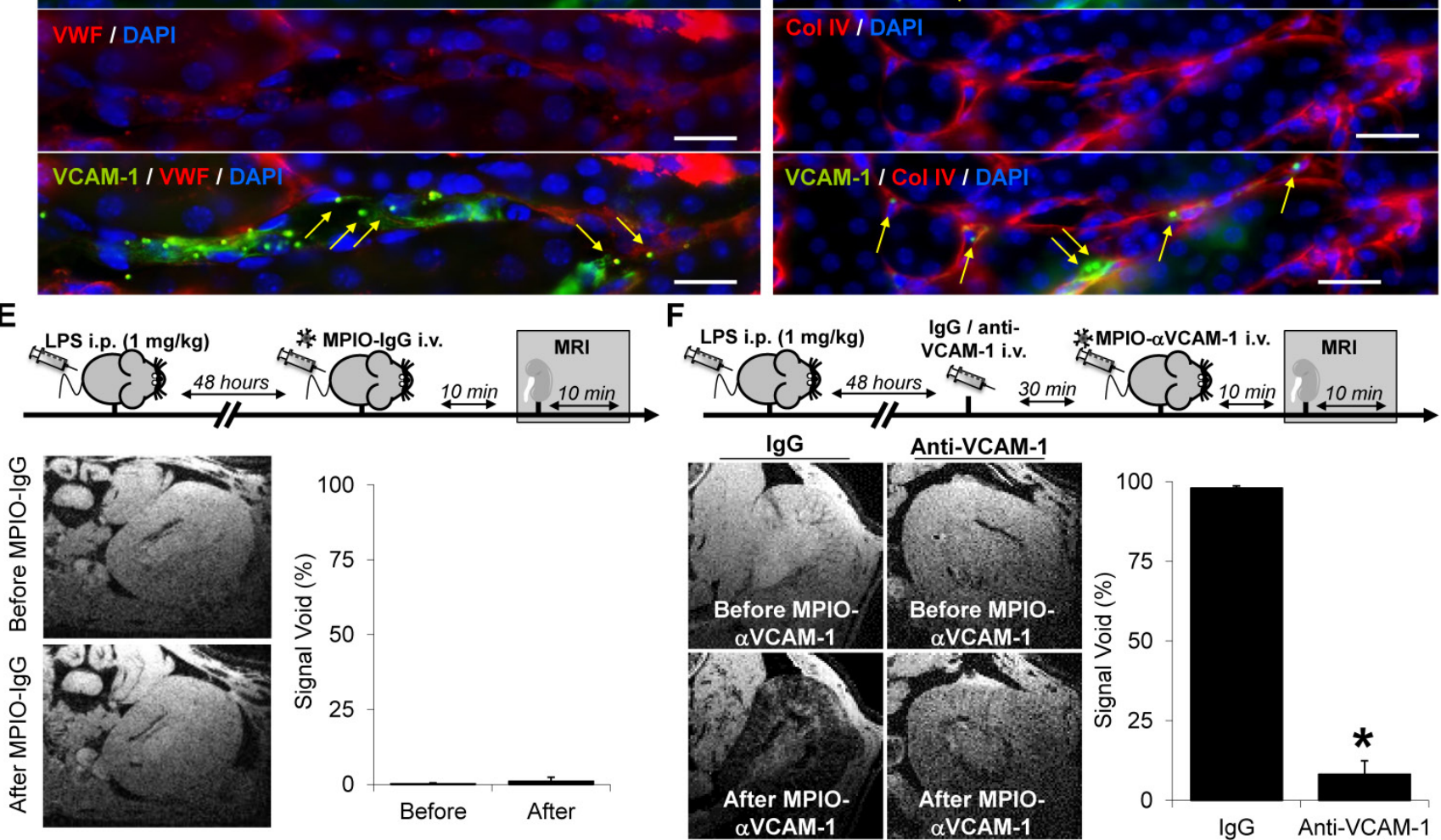

Figure 1. MPIO- $\alpha$ VCAM-1 enhanced MRI reveals endothelial activation in the kidney during systemic inflammation. (A) Schematic representation of the experimental design. (B) Top, Representative consecutive slices (from a single mouse) from T2-weighted images of the kidney from LPS-treated mice (1 mg/kg) revealing no apparent abnormality. Middle, representative T2*-weighted images of the kidney from LPS-treated mice $(1 \mathrm{mg} / \mathrm{kg})$ before MPIO- $\alpha$ VCAM-1 administration. Bottom, same as middle but after MPIO- $\alpha$ VCAM-1 administration, revealing numerous confluent signal voids in both kidneys (dark areas). (C) Top, Representative consecutive slices (from a single mouse) from T2*-weighted images from control and LPS-treated mice (1 mg/kg) after MPIO- $\alpha$ VCAM-1 administration. Bottom, corresponding quantification ( $n=3$ /group). ${ }^{*} p<0.05$ vs control/saline. (D) Immunohistological images of MPIO- $\alpha$ VCAM-1 in kidneys from LPS-treated mice. Green represents VCAM-1, Red represents collagen type IV or von Willebrand factor (both used as endothelial markers) and Blue represents DAPI. The yellow arrows indicate MPIO- $\alpha$ VCAM-1 localization (autofluorescent green dots). (E) Top: Experimental design. Left: Representative T2* weighted images of the right kidney of LPS-treated mice before (top) and after (bottom) MPIO-IgG injection. Right: Corresponding quantification ( $\mathrm{n}=4 /$ group). $n s=$ non-significant $(\mathrm{F})$ Top: Experimental design. Left: Representative T2* weighted images of the right kidney of LPS-treated mice before (top) and after (bottom) MPIO- $\alpha$ VCAM-1 injection. All mice were pre-treated with either control lgG or anti-VCAM-1 lgG (2.5 mg/kg, competition experiment). Right: Corresponding quantification ( $\mathrm{n}=5 / \mathrm{group})$. 

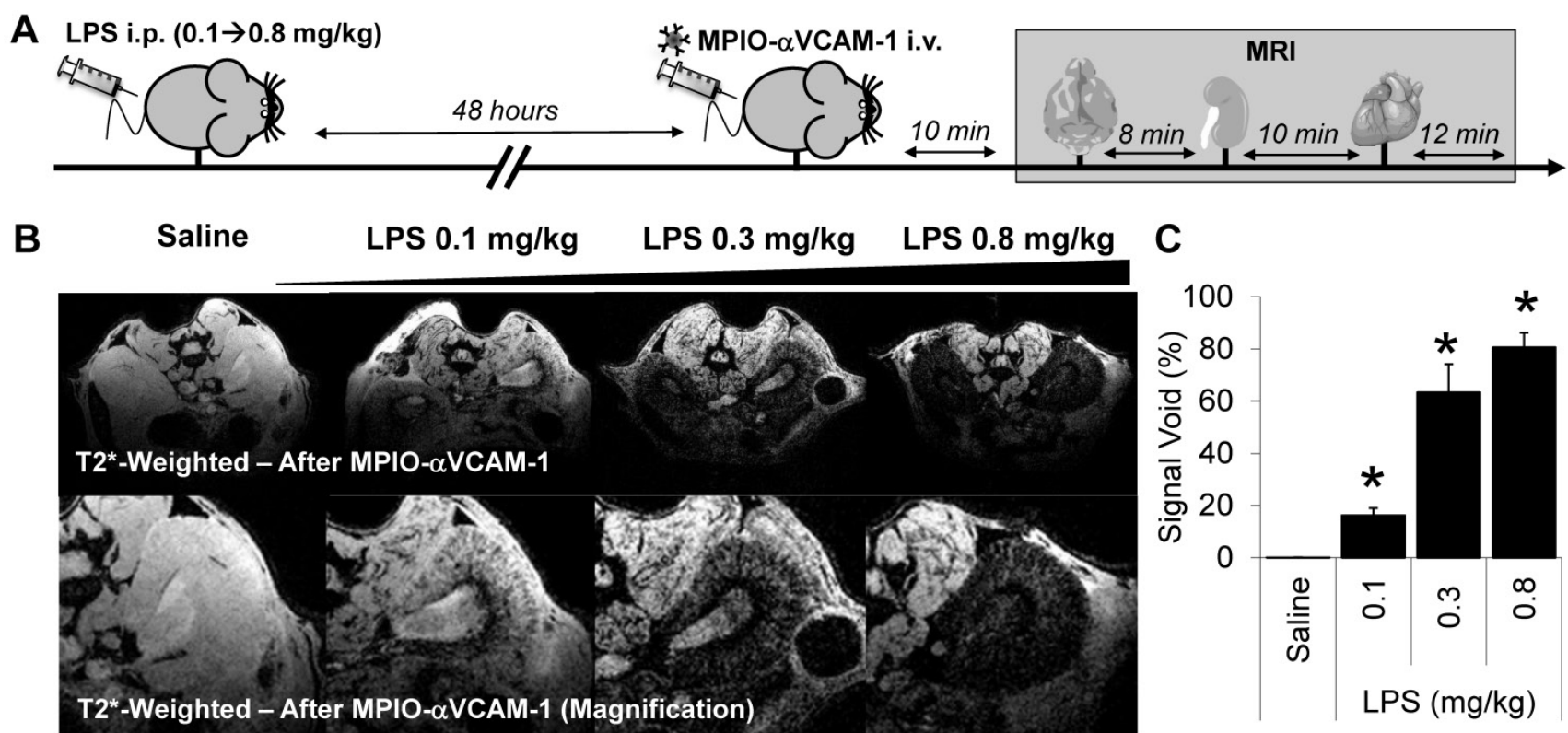

D

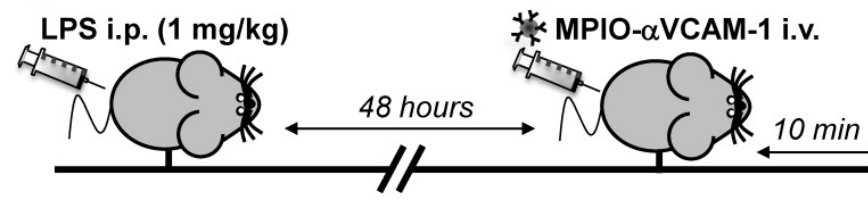

E

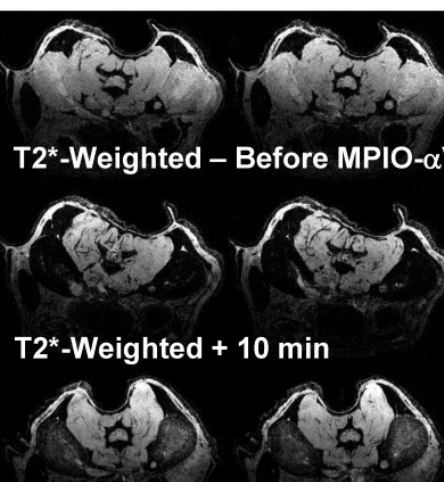

T2* -Weighted + 2 hours
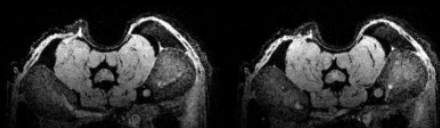

T2*-Weighted + 4 hours
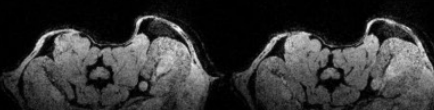

T2*-Weighted +24 hours

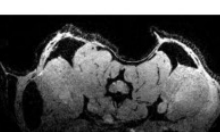

CAM-1
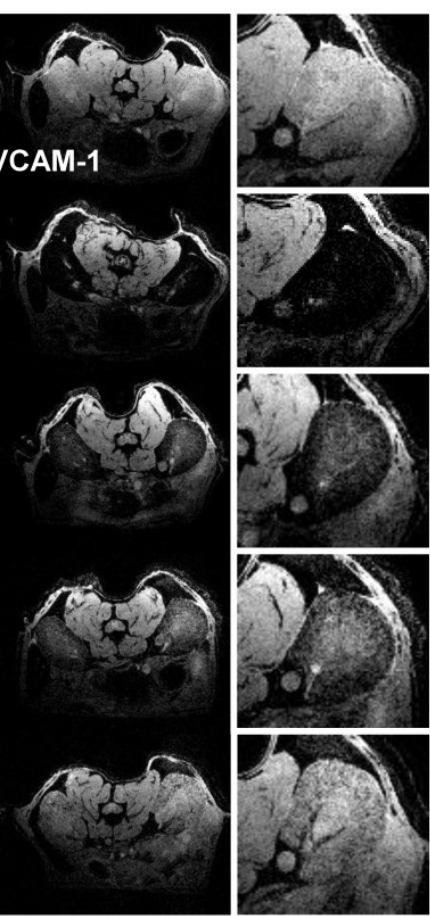

F 0
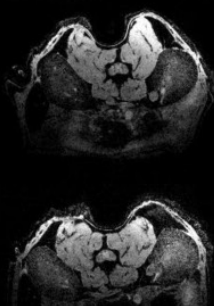

+2 hours

Before

$+10 \min$

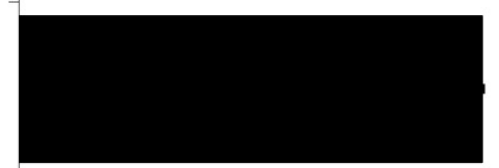

Signal Void (\%)

50

100

Figure 2. MPIO- $\alpha$ VCAM-1 enhanced MRI allows semi-quantitative measurement of endothelial activation in the kidneys with a temporal resolution of 24 hours. (A) Experimental design. (B) Representative high-resolution T2*-weighted images without (top) and with magnification (bottom) from mice treated with either saline or different amounts of LPS (from 0.1 to $0.8 \mathrm{mg} / \mathrm{kg}$ ). (C) Corresponding quantification ( $\mathrm{n}=3 /$ group). ${ }^{*} \mathrm{p}<0.05$ vs control/saline. (D) Experimental design. (E) Representative consecutive slices (from a single mouse) from high-resolution T2*-weighted images before and at different time after MPIO-aVCAM-1 administration in LPS mice (1 mg/kg). This allows to appreciate the kinetic of MPIO- $\alpha$ VCAM-1 clearance from the inflamed kidneys 48 hours after LPS administration. Signal voids decreased gradually with time after MPIOs- $\alpha$ VCAM-1 injection. (F) Corresponding quantification ( $n=3 /$ group). 

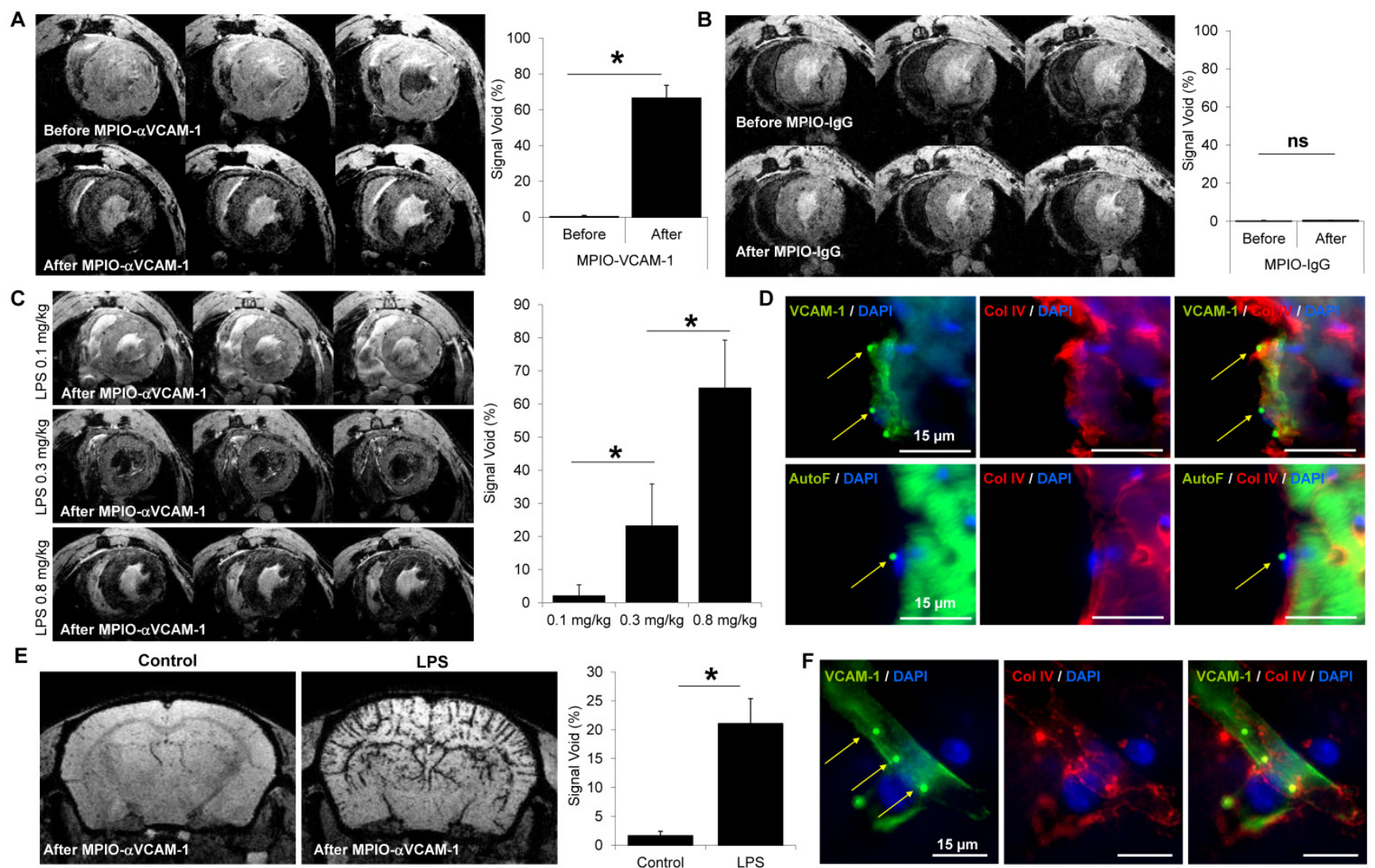

Figure 3. A single MPIO- $\alpha$ VCAM-I injection allows multi-organ mapping of endothelial activation: examples of the brain and the heart. (A) Left: Representative consecutive slices (from a single mouse) from T2*-weighted images of the heart from LPS-treated mice (1 mg/kg) before (top) and after (bottom) MPIO- $\alpha$ VCAM- 1 administration. Right: Corresponding quantification ( $n=4 /$ group). (B) Left: Representative consecutive slices (from a single mouse) from T2* weighted images of the heart of LPS-treated mice before (top) and after (bottom) MPIO-IgG injection. Right: Corresponding quantification ( $\mathrm{n}=4$ /group). (C) Left: Representative consecutive slices (from a single mouse) from T2*-weighted images of the heart from LPS-treated animals at different weight-adjusted doses $(0.1,0.3 \mathrm{and} 0.8 \mathrm{mg} / \mathrm{kg}) 20 \mathrm{minutes}$ after MPIO- $\alpha$ VCAM-1 injection. Right: Corresponding quantification ( $n=4 /$ group). ${ }^{*} \mathrm{p}<0.05$ vs lower dose. (D) Representative images of MPIO- $\alpha$ VCAM-1 inside the endocardium (Top) or VCAM- $1+$ vessels (Bottom) of the heart as seen by immunofluorescence. The yellow arrows indicate MPIO- $\alpha$ VCAM-1 localization (MPIOs are fluorescent in green). (E) Left: Representative T2*-weighted images of the brain from control and LPS-treated mice $(1 \mathrm{mg} / \mathrm{kg}$ ) after MPIO- $\alpha$ VCAM-1 administration. Right: Corresponding quantification ( $\mathrm{n}=3 / \mathrm{group})$. ( $\mathrm{F}$ ) Representative images of MPIO- $\alpha$ VCAM-1 inside a VCAM-1+ vessel of the brain as seen by immunofluorescence.

In the brain, we already demonstrated the sensitivity and specificity of MPIO- $\alpha$ VCAM-1 enhanced MRI to detect endothelial cell activation triggered by neuroinflammation in a previous study[18]. Here, our aim was to confirm these previous results in the LPS model in the context of multi-organ imaging. As shown on Fig. 3E, MPIO- $\alpha$ VCAM-1 enhanced MRI revealed endothelial activation in the brain 48 hours after LPS administration, in line with our immunohistological analyses. After MPIO- $\alpha$ VCAM-1 administration, numerous MPIO were revealed in the lumen of VCAM-1+ vessels in the brain on histological analysis (Fig. 3F). This demonstrates that a single injection of MPIO- $\alpha$ VCAM-1 allows fast, sensitive, specific, semi-quantitative, longitudinal and multi-organ mapping of endothelial activation after LPS administration.

\section{Mapping endothelial activation during renal insufficiency}

Kidney is an organ of major interest when dealing with cardiovascular diseases, especially because chronic kidney disease increases the risk of cardiovascular events. Moreover in various clinical situations, and both as cause or consequence, kidney failure is associated with heart failure. Thus we wanted to evaluate endothelial activation in the cardiovascular system in models of acute and chronic renal insufficiency.

To induce AKI, we chose a model of rhabdomyolysis since it is a frequent clinical situation and does not involve invasive surgical procedures, which could be associated with unspecific endothelial activation. In this model, an intramuscular injection of glycerol is performed in the two limbs to induce rhabdomyolysis, thereby releasing myoglobin from the muscles into the bloodstream and leading to subsequent AKI (related to hypovolemia and direct toxicity of myoglobin on renal tubules). In our hands, two days after the intramuscular injection of glycerol, an AKI was observed, as assessed by the significant raises in creatinine and urea (BUN) plasmatic levels (Fig. S7A-B).

In this model, as shown on Fig. 4A-C, overexpression of VCAM-1 was detected by histological 
staining in both medullar and cortical regions of the kidney (this VCAM-1 overexpression was extra-glomerular, Fig. S8). According to these results, MPIO- $\alpha$ VCAM-1 enhanced T2*-weighted MRI of the kidneys showed a significant increase in signal void in glycerol-treated animals two days after injection (Fig. 5D and E). Interestingly, rhabdomyolysis-induced AKI was transient and kidney almost recovered a normal function 8 days after glycerol injection (Fig. S7C). Consistently, MPIO- $\alpha$ VCAM-1 enhanced MRI indicated a decrease in the intensity of endothelial activation, coherent with the repair phase (Fig. 4F). Of note, whereas kidney function was almost back to normal (as assessed by creatinine and urea plasmatic levels), VCAM-1 expression was still clearly detectable at 8 days, suggesting persistent, sub-clinical endothelial activation in the kidneys that lasts for days after an episode of AKI.

In contrast to the results obtained for the kidneys, VCAM-1 was not detected by immunohistological analyses in the brain of mice subjected to rhabdomyolysis, neither in the hippocampus nor in the cortex (Fig. 5A-B). In line with these immunohistological analyses, MPIO- $\alpha$ VCAM-1 enhanced MRI showed no VCAM-1 overexpression in the brain (Fig. 5C). Similar results were obtained for the heart (data not shown). Thus, in this model of acute renal insufficiency, endothelial activation occurred exclusively in the kidney, revealing an organ-specific rather than a systemic endothelial activation state.

We also investigated whether chronic renal insufficiency influenced the endothelial activation status in the target organs. Thus, we investigated whether 8 weeks of stable chronic kidney disease (i.e. without ongoing kidney injury) was accompanied by endothelial cell activation, as assessed by MPIO- $\alpha$ VCAM- 1 enhanced MRI. To this aim, we injected MPIO- $\alpha$ VCAM-1 in subtotal nephrectomized mice (SNX), 8 weeks after surgery (Fig. 5D). Although those mice exhibited elevated urea and creatinine levels, MPIO- $\alpha$ VCAM- 1 did not reveal any endothelial activation in the kidneys (Fig. 5E-F). The same experiments in the brain and heart also failed to demonstrate endothelial activation (data not shown). Thus, in this case of isolated and stable chronic kidney disease, endothelial cells of the microvasculature of the target organs remain quiescent (Fig. 5G).

\section{Mapping endothelial cell activation in diabetes mellitus}

We also performed multi-organ mapping of endothelial activation in a model of diabetes mellitus. Chronic hyperglycemia in diabetes mellitus is known to induce numerous chronic complications including micro- and macrovascular injuries, whose diagnosis is often hampered by silent or minor clinical presentation. We performed multi-organ MRI after injection of MPIO- $\alpha$ VCAM-1 in diabetic mice (streptozotocin model) after 4 weeks of disease evolution.

Endothelial activation level significantly differed in the three vascular beds investigated. Indeed, 4 weeks after diabetes induction (STZ-treated mice), MPIO- $\alpha$ VCAM-1 MRI revealed significant endothelial activation in the kidneys of diabetic mice as compared to controls (Fig. 6A-B). This endothelial activation was confirmed by immunohistochemistry in the diabetic mice group (Fig. 6C). In contrast, no endothelial activation was detected in the brain of the diabetic mice (Fig. 6D). Interestingly enough, in the heart, MPIO- $\alpha$ VCAM-1 enhanced MRI revealed VCAM-1 overexpression selectively in the endocardium of STZ-treated mice (Fig. 6E). These data in the three vascular beds demonstrated that diabetic mice present sustained endothelial activation in the kidneys and the heart, the two main target organs of diabetes.

\section{Mapping endothelial cell activation in normal aging}

Lastly, we performed endothelial activation mapping in aging mice. Indeed, recent studies demonstrated that aging is associated with a chronic inflammatory state (known as inflammaging), characterized by elevated levels of Interleukin-1, Interleukin-6 and Tumor Necrosis Factor in the plasma.[21] These data suggest that aging may be associated with a chronic endothelial activation state. To test this hypothesis, we performed endothelial activation mapping in naive 3-month-old and 18-month-old mice, breaded under the same conditions. As shown on Fig. 7A-C, endothelial activation was significantly stronger in old than in young mice in the kidney. Similarly, but with a lower level of VCAM-1 overexpression, the cerebrovasculature appeared activated in the brain of aged mice (Fig. 7D-E). In contrast, no difference was observed in the heart (data not shown). These results demonstrate that aging is associated with an elevated level of endothelial activation in the kidney, and to a lesser extent, in the brain (Fig. 7F).

\section{Discussion}

Altogether, our results demonstrate the feasibility of endothelial activation mapping in multiple organs using a single injection of MPIO- $\alpha$ VCAM- 1 and MRI (Fig. 8). This molecular imaging method allows, for the first time, evaluation of the endothelial activation status in all the studied organs in a single experiment and in a timely manner. Using this method, we uncovered chronic endothelial activation in the heart and kidneys of diabetic mice. Moreover, our data revealed abnormal chronic activation of the kidney and brain endothelia in aged mice. 

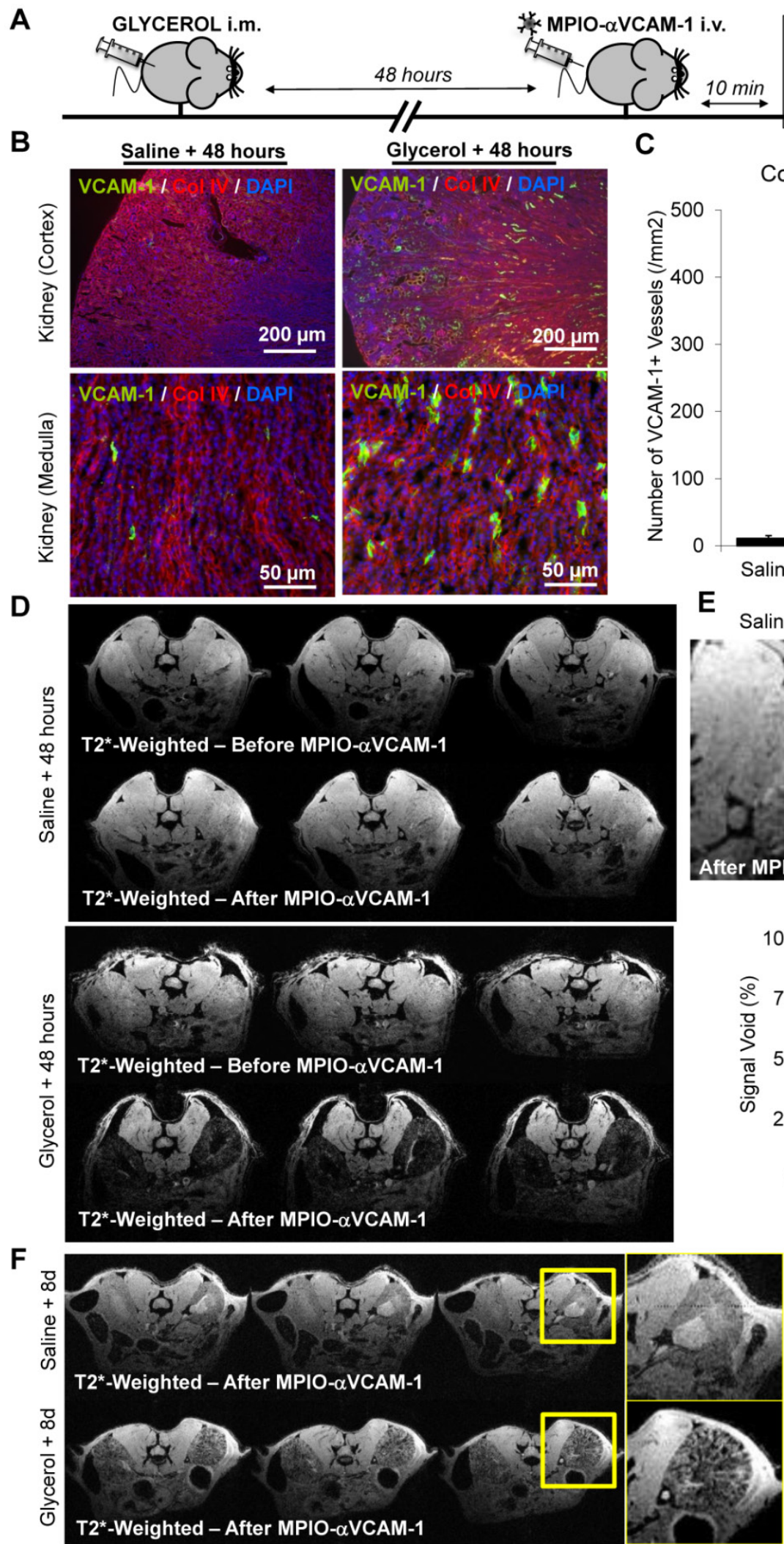

Cortex

Medulla

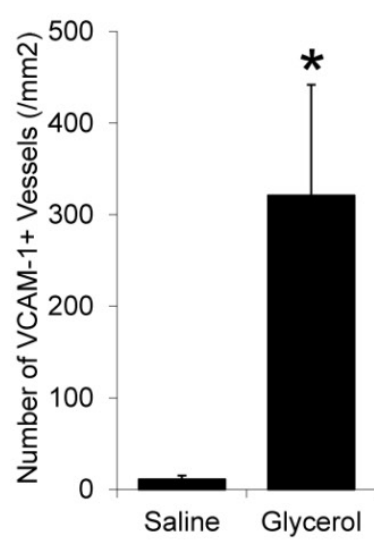

E
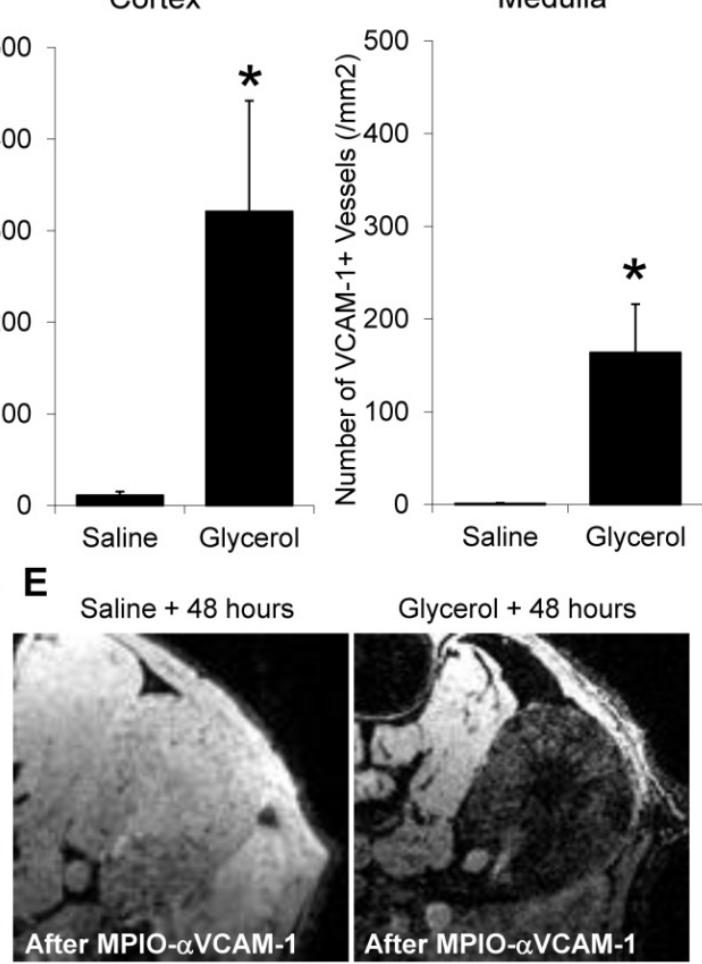

Glycerol + 48 hours

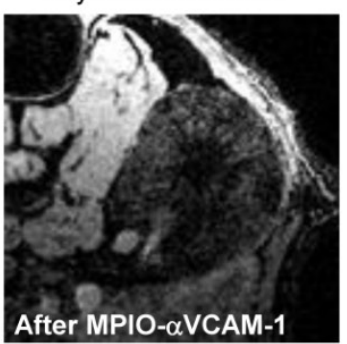

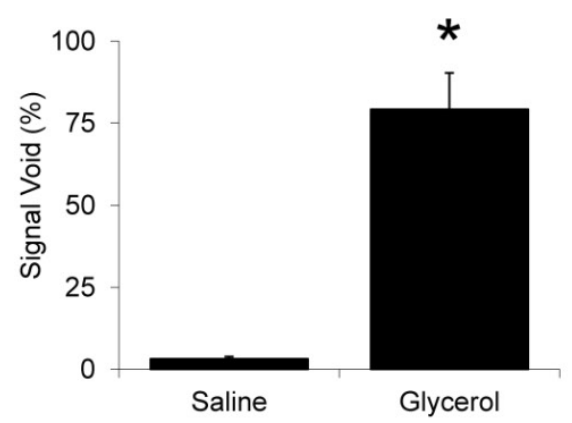

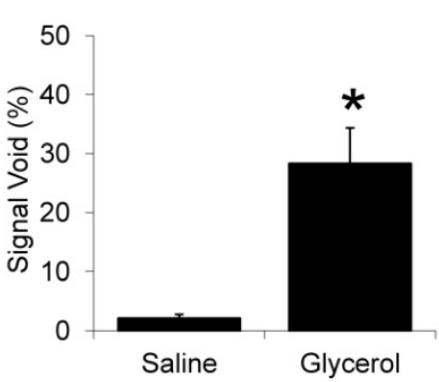

Figure 4. MPIO- $\alpha$ VCAM-I enhanced MRI reveals selective endothelial activation in the kidneys induced by rhabdomyolysis. (A) Experimental design. (B) Representative immunohistological images of VCAM-1 expression (Green) in the kidney (cortex and medulla) 2 days (2d) after saline (left) or glycerol (right) injection. Collagen type IV (Red) and DAPI (Blue) were used to evaluate renal tissue morphology. VCAM-1 was overexpressed in the medulla and cortex of glycerol-treated mice as compared to controls (saline). (C) Corresponding quantification in the cortex and medulla of the kidneys ( $\mathrm{n}=5 /$ group). * $<0.05$ vs control (saline). (D) Representative consecutive slices (from a single mouse) from T2*-weighted images of the kidneys from saline (top) or glycerol (bottom) treated mice before and after MPIO- $\alpha$ VCAM-1 administration. (E) Top: Representative high-magnification T2*-weighted images of saline- and glycerol-treated mice after MPIO- $\alpha$ VCAM-1 administration 2 days (2d) after injections. Bottom: Corresponding quantification ( $n=5 /$ group). ${ }^{*} \mathrm{p}<0.05$ vs control (saline). ( $F$ ) Left: Representative consecutive slices (from a single mouse) from T2*-weighted images after MPIO- $\alpha$ VCAM-1 administration 8 days after saline (top) or glycerol (bottom) injections. Yellow squares indicate magnification areas. Right: Corresponding quantification ( $n=5$ /group). ${ }^{*} \mathrm{p}<0.05$ vs control (saline). 
A

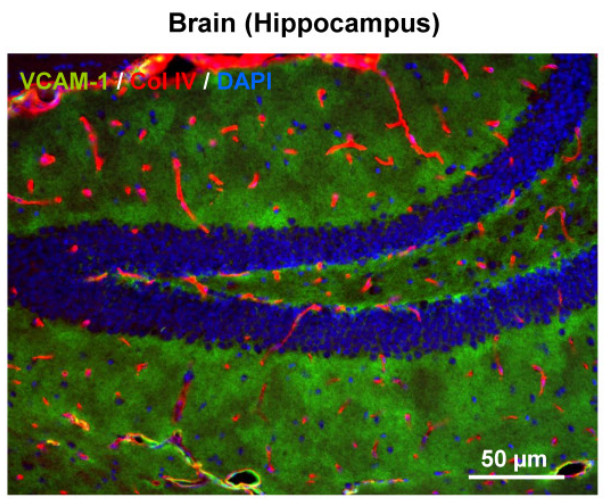

B

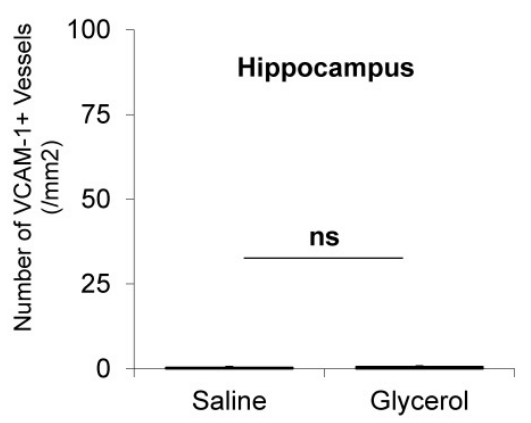

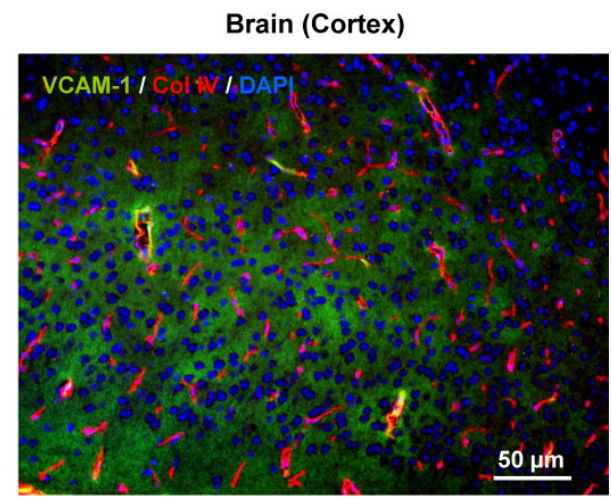

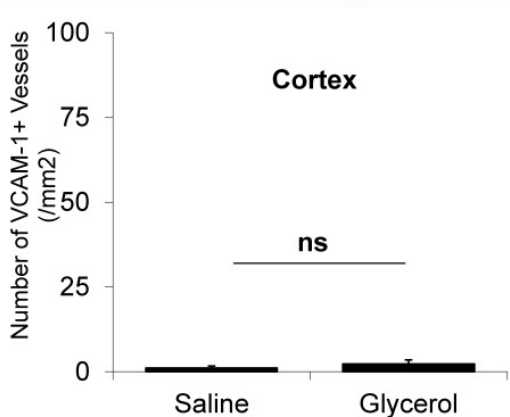

C
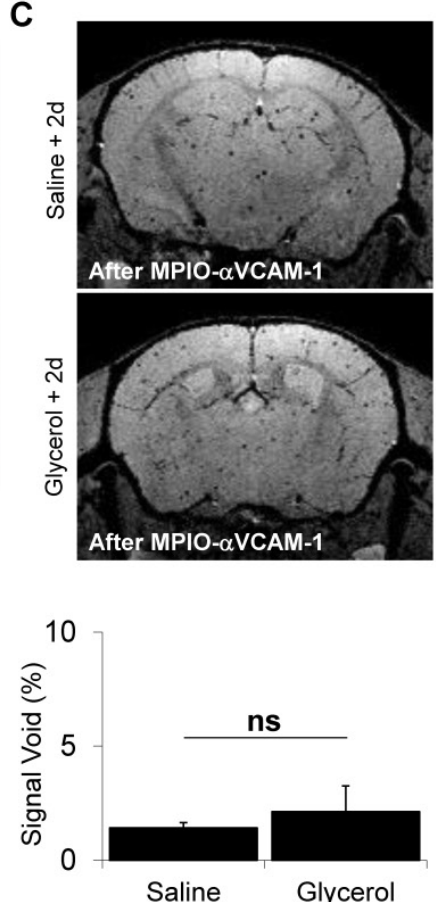

D Subtotal Nephrectomy (SNX)

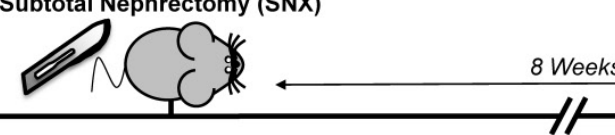

E

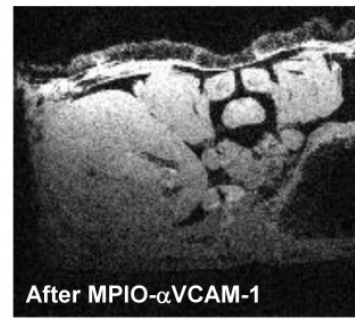

Sham

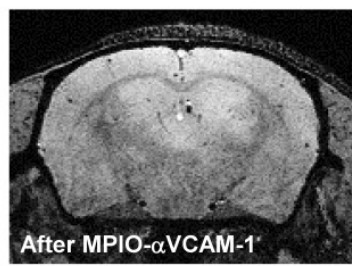

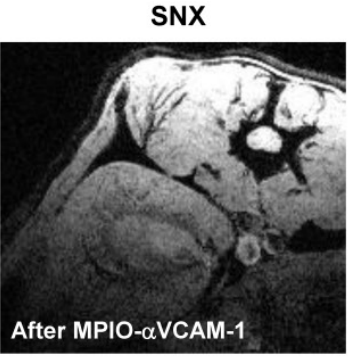

SNX

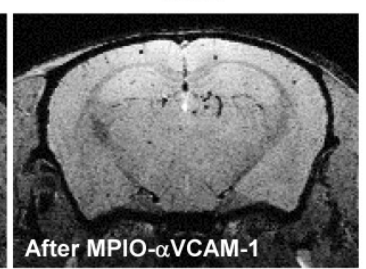

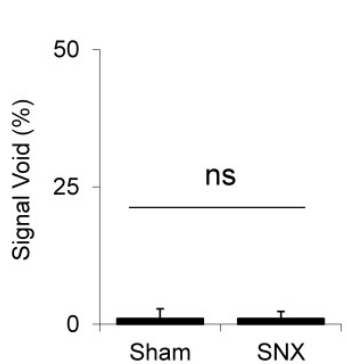

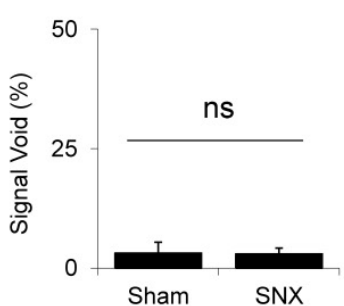

落 MPIO- $\alpha$ VCAM-1 i.v.
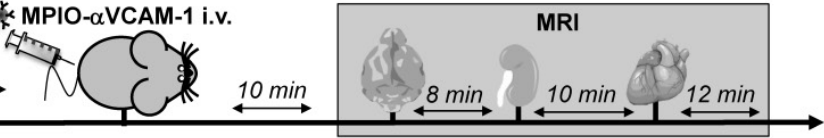

$\mathbf{F}$

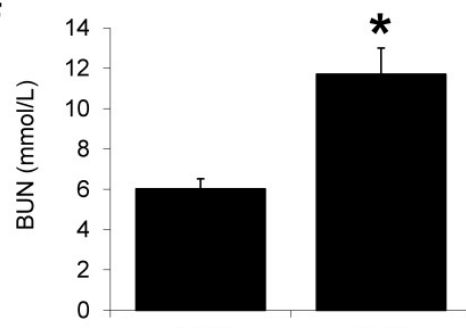

G

Sham

SNX
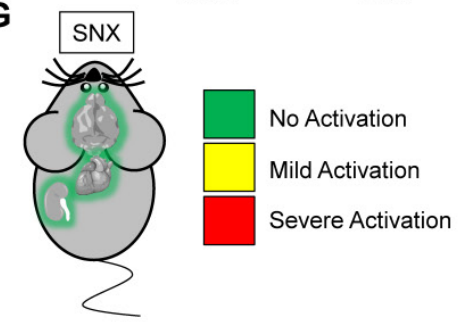

Figure 5. Acute or chronic renal insufficiencies do not promote endothelial activation in distal organs. (A) Representative immunohistological VCAM-1 images of the brain hippocampus and cortex 48 hours after glycerol injection. Collagen type IV (Red) and DAPI (Blue) were used to assess brain tissue morphology. (B) Corresponding quantifications, demonstrating the lack of VCAM-1 upregulation in the brain (neither in the hippocampus nor cortex) after rhabdomyolysis (glycerol treatment), as compared to controls (saline), ( $n=5 / g r o u p)$. ns=non significative differences were observed. (C) Top: Representative T2*-weighted images of the brain from control (saline) and glycerol-treated mice after MPIO- $\alpha$ VCAM-1 administration, 48 hours after rhabdomyolysis induction. Bottom: Corresponding quantification ( $n=5 / g r o u p)$. (D) Experimental design. (E) Left: Representative T2*-weighted images of the kidneys and brain of control (Sham) and subtotal nephrectomized mice (SNX), after MPIO- $\alpha$ VCAM-1 administration. Right: Corresponding quantifications ( $n=5 /$ group). (F) Kidney function assessed by BUN levels ( $n=6 /$ group). (G) Schematic representation of the results. * $p<0.05$ vs control (Sham). ns= non significant differences were observed. 
A $5 \times$ STREPTOZOTOCIN i.p. (STZ, $50 \mathrm{mg} / \mathrm{kg}$ )
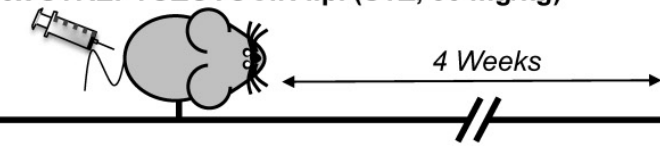

MPIO- $\alpha$ VCAM-1 i.v.

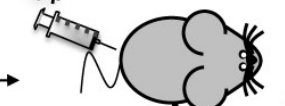

$\stackrel{\mathrm{min}}{\longleftrightarrow}$

B

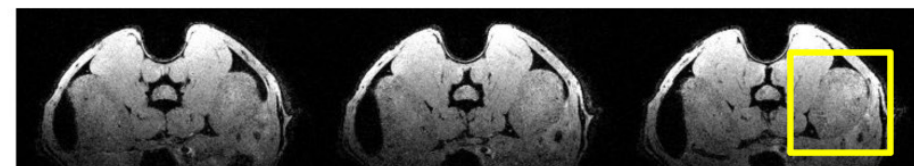

Control
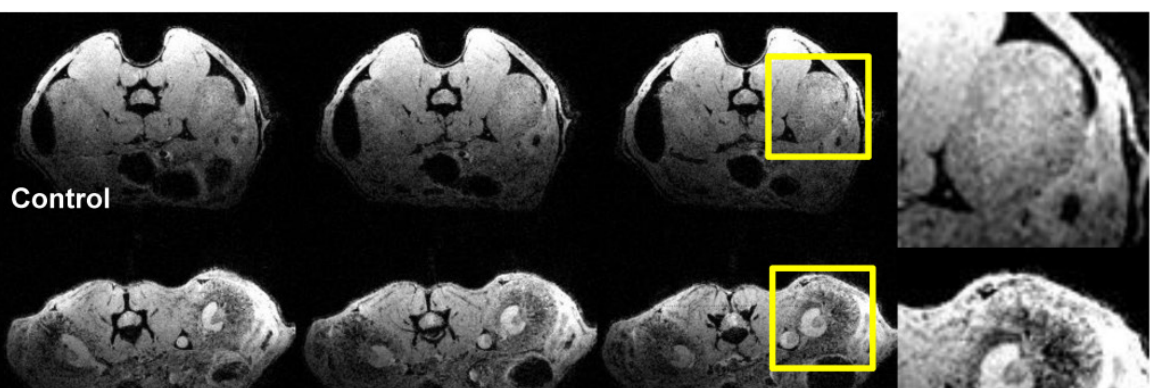

\section{STZ-Treated}

VCAM-1 / COITVI DAPI
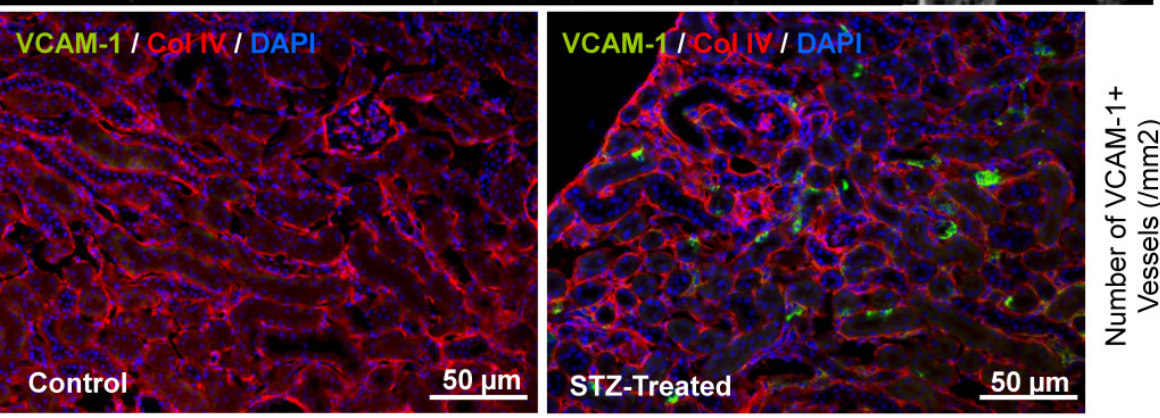

D
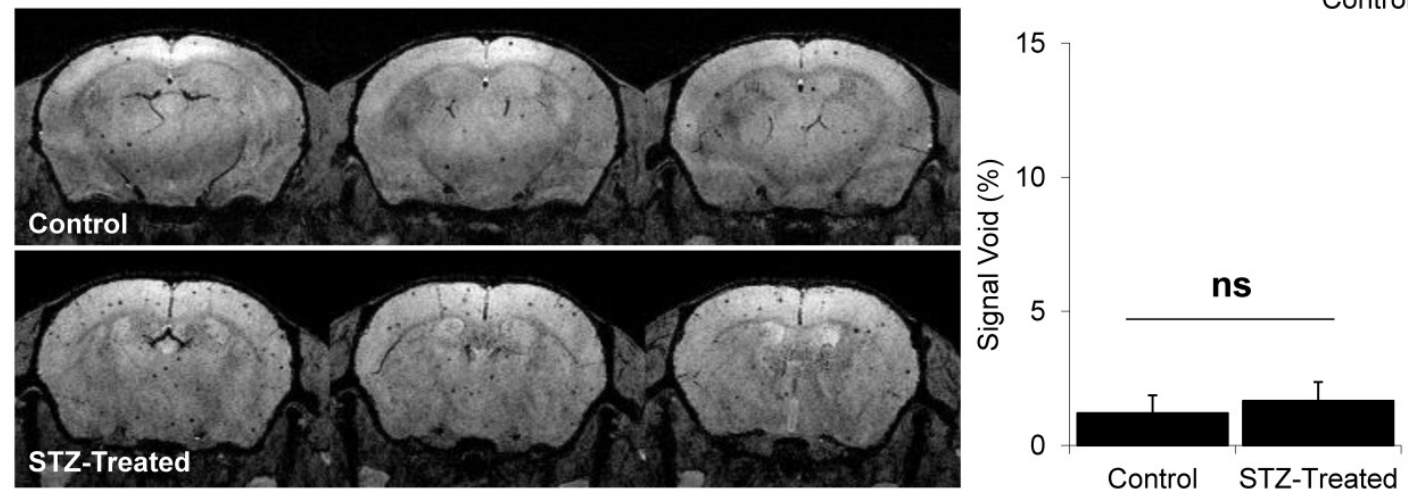

E
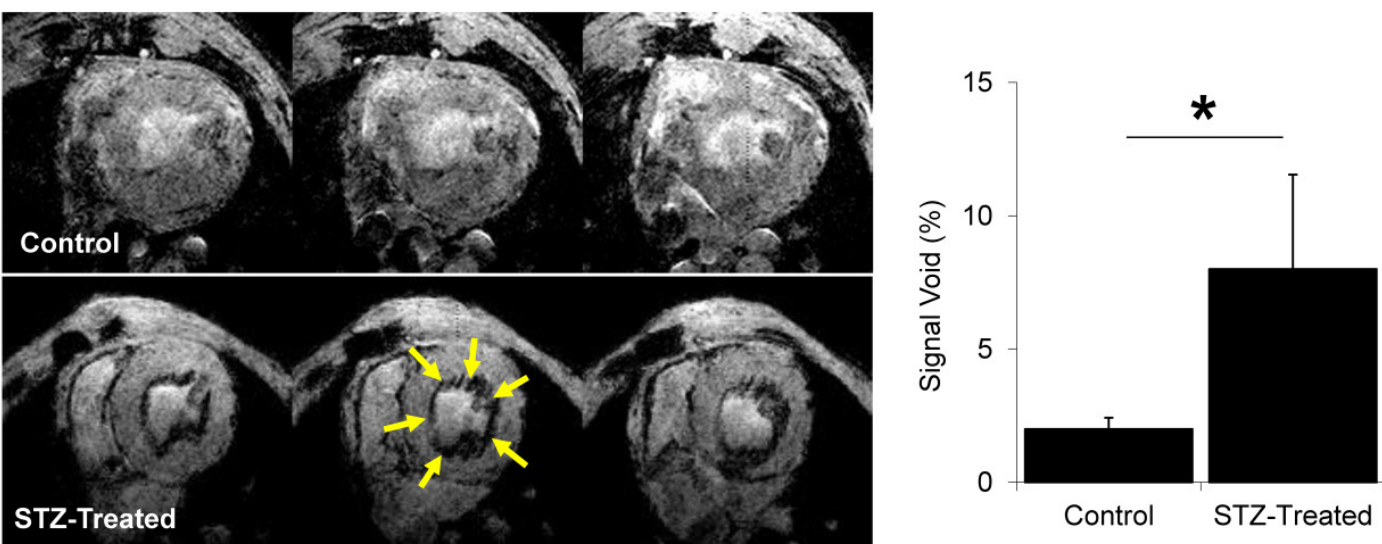

Figure 6. Endothelial activation mapping reveals kidney and endomyocardial inflammation in diabetes mellitus. (A) Experimental design. (B) Left: Representative consecutive slices (from a single mouse) from T2*-weighted images of kidneys from control and STZ-treated mice, after MPIO- $\alpha$ VCAM-1 administration. Yellow squares indicate magnification areas. Right: Corresponding quantification ( $n=5 /$ group). (C) Left: Representative immunohistological images of VCAM-1 expression (Green) in the kidneys of saline and STZ-treated mice 8 weeks after injection. Right: Corresponding quantification ( $n=5 /$ group). Collagen type IV (Red) and DAPI (Blue) were used to assess renal tissue morphology, which was similar in the two groups. (D) Left: Representative consecutive slices (from a single mouse) from T2*-weighted images of the brains from control and STZ-treated mice after MPIO- $\alpha$ VCAM-1 administration. Right: Corresponding quantification ( $n=5 / g r o u p)$. (E) Left: Representative consecutive slices (from a single mouse) from T2*-weighted images of the hearts from control and STZ-treated mice after MPIO- $\alpha$ VCAM-1 administration. Yellow arrows indicate MPIO- $\alpha$ VCAM- 1 induced signal voids in the endocardium of STZ-treated mice. Right: Corresponding quantification ( $n=5 /$ group). * $p<0.05$ vs control/saline. 


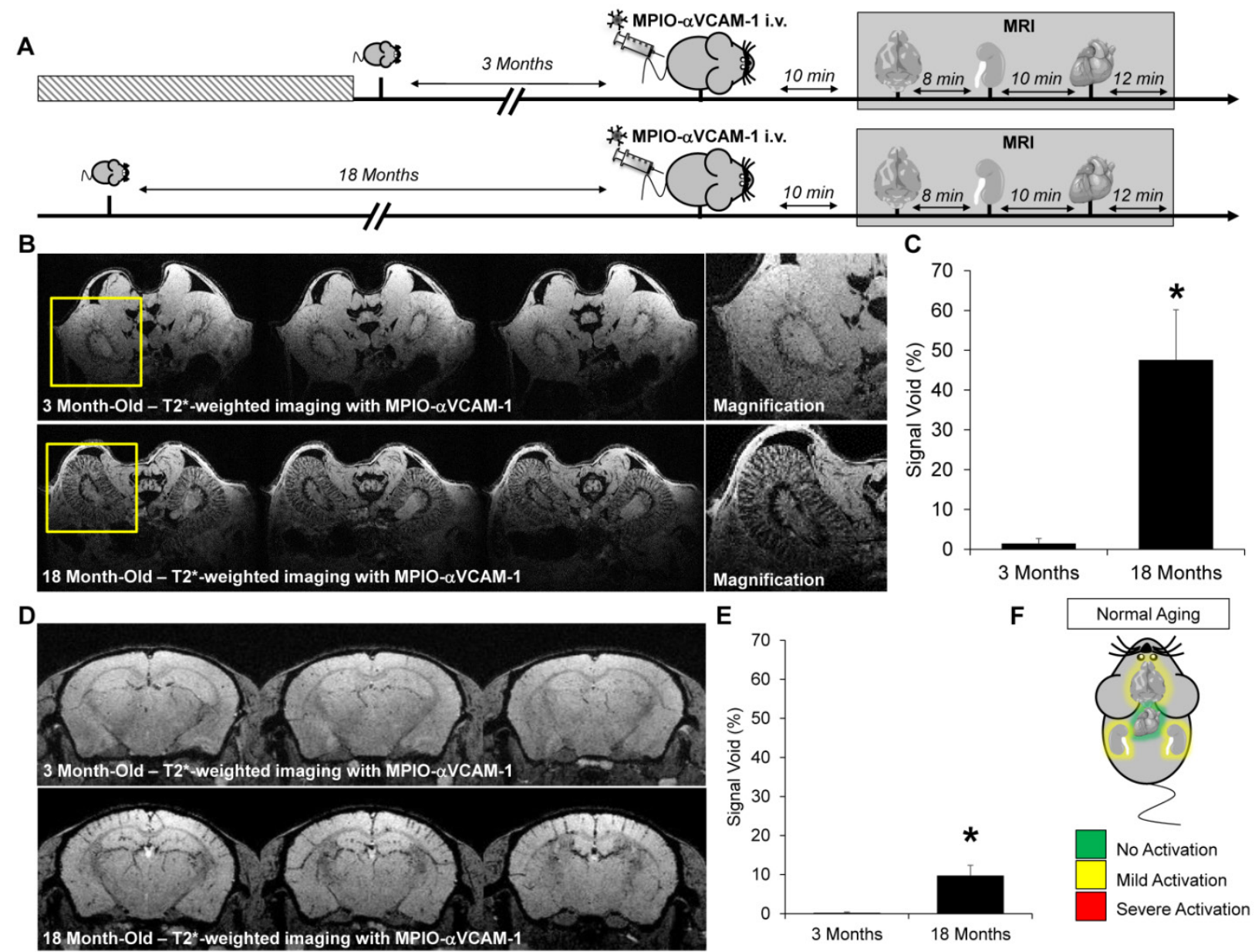

Figure 7. Endothelial activation mapping unmasks kidney and cerebral inflammation in aged mice. (A) Experimental design. (B) Representative consecutive slices (from a single mouse) from T2*-weighted images of kidneys from young ( 3 month-old) and aged (18 month-old) mice, after MPIO- $\alpha$ VCAM-1 administration. Yellow squares indicate magnification areas. (C) Corresponding quantification ( $n=4 /$ group). (D) Representative consecutive slices (from a single mouse) from T2*-weighted images of the brain from young ( 3 month-old) and aged (18 month-old) mice, after MPIO- $\alpha$ VCAM-1 administration. (E) Corresponding quantification ( $n=4 / g r o u p)$. (F) Schematic representation of the findings in aged mice. ${ }^{*} \mathrm{p}<0.05$ vs young mice.

Among the proteins overexpressed by activated endothelium, VCAM-1 was selected since it has unique properties for highly sensitive molecular imaging. Indeed, VCAM-1 is not constitutively expressed by quiescent endothelium but is strongly upregulated in inflammatory conditions.[4] Thus, the lack of VCAM-1 expression by quiescent endothelium ensures imaging specificity, whereas the strong and steady VCAM-1 expression by activated endothelium allows high imaging sensitivity. Moreover, the present method allows imaging of the earliest step of the inflammatory reaction, which represents a significant advantage over cell or platelet-targeted molecular probes. $[22,23]$ Of note, binding of MPIOs to VCAM-1 on the endothelium does not trigger further endothelial inflammation by itself.[4] The high specificity demonstrated in the present study also highlights the potential of micron-sized particles to specifically target activated endothelial cells for the controlled release of anti-inflammatory or immune-suppressive drugs.
Our results in diabetic and aged mice further support a critical role for endothelial activation in the higher prevalence of cardiovascular diseases among aged and diabetic individuals. In both conditions, endothelial activation mapping revealed the kidney endothelium as the most affected microvasculature. These results should be considered in light of the high prevalence of chronic kidney diseases in diabetic and aged patients. The lack of effect of chronic kidney disease by itself on the activation state of endothelia from the cardiovascular system was more surprising. Indeed, kidneys are thought to mediate clearance of pro-inflammatory byproducts of the metabolism. Accordingly, there is a strong correlation between inflammatory biomarkers and kidney function in humans, especially in the elderly.[24] It is plausible that the levels of renal insufficiency achieved by subtotal nephrectomy or rhabdomyolysis could promote systemic inflammation triggered by another injury. However, they may not be sufficient alone to drive systemic inflammation and endothelial activation. 
A

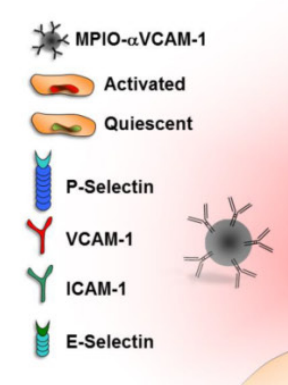

C
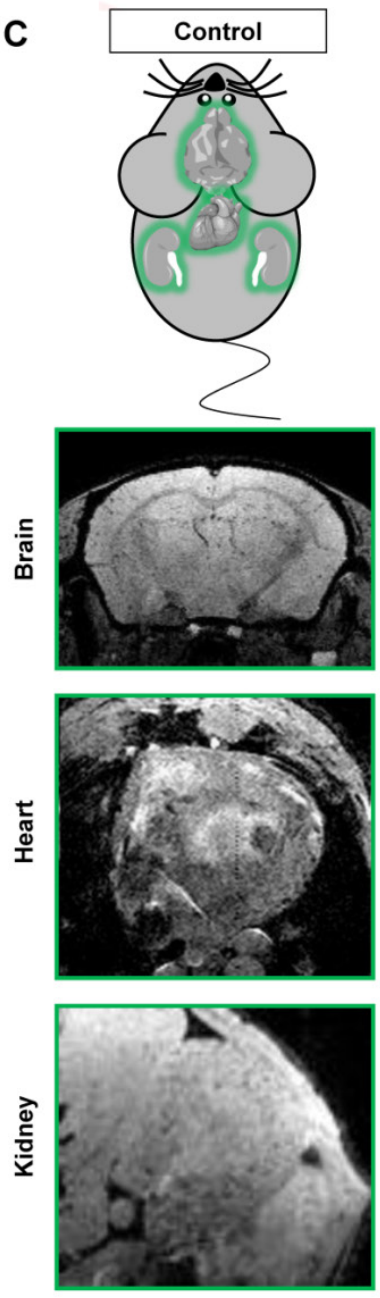

B
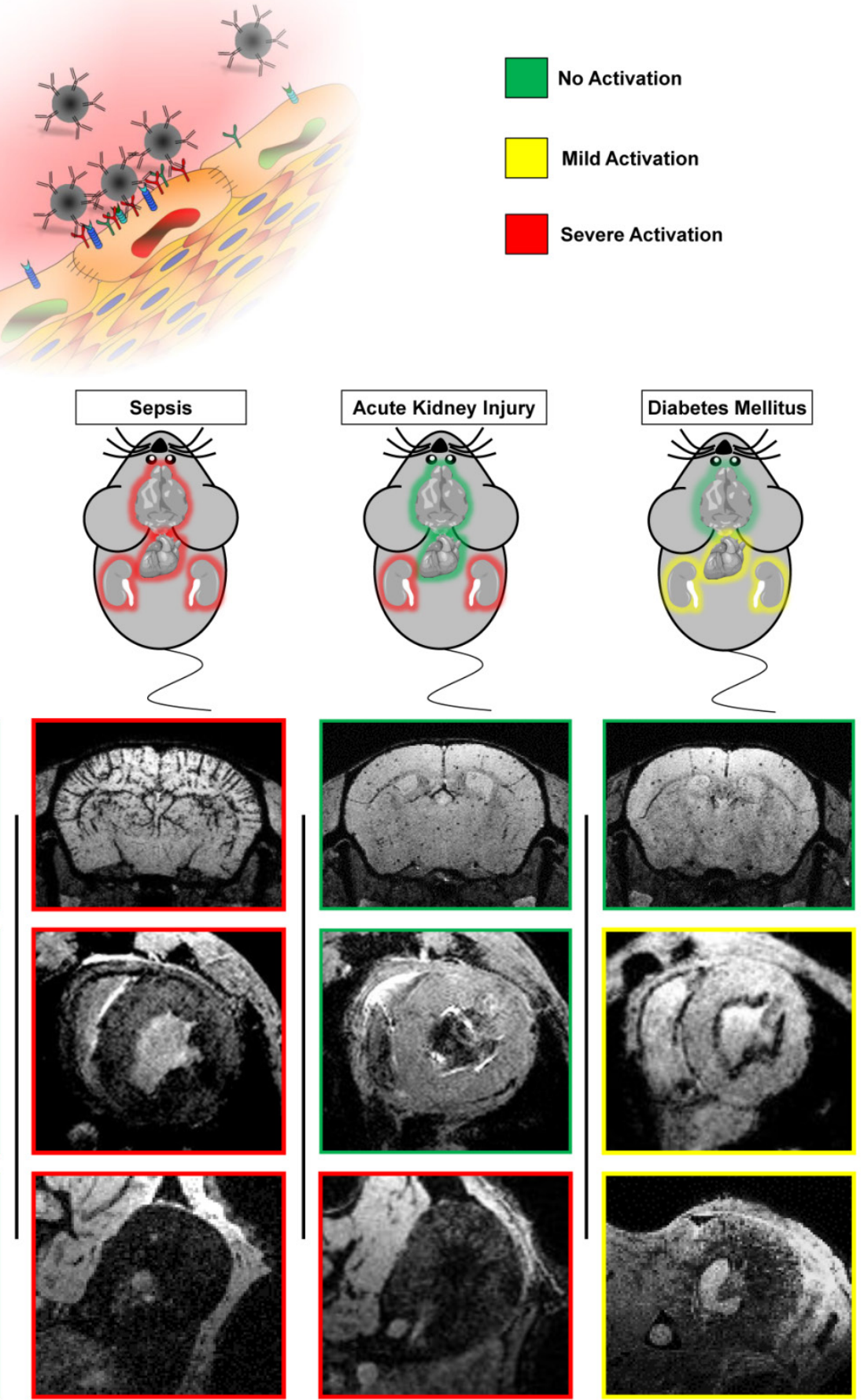

No Activation

Mild Activation

Severe Activation
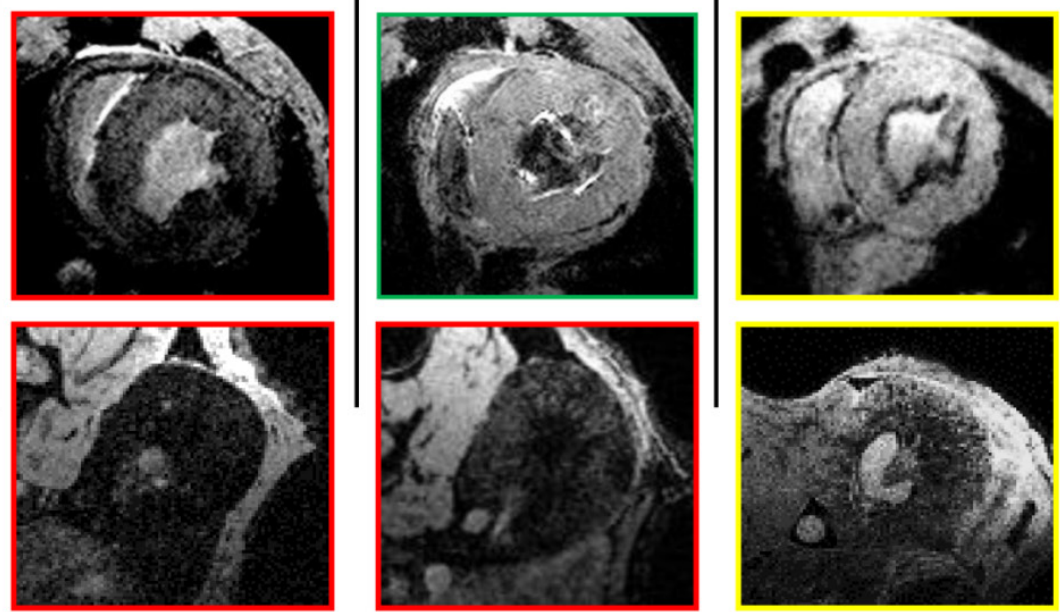

Figure 8. Schematic representation of the main findings. (A) Schematic representation of the binding of circulating MPIO- $\alpha$ VCAM- 1 selectively to activated endothelial cells expressing VCAM-1. Whereas other adhesion molecules are also expressed by quiescent endothelial cells, VCAM-1 is only expressed after activation. Therefore, MPIO- $\alpha$ VCAM-1 are specific for activated endothelial cells. (B) Figure legend. (C) Representative images and schematic representation of the main findings.

Importantly, some of our results concerning the patterns of VCAM-1 expression in the different experimental models performed are supported by the literature. For instance, overexpression of adhesion molecules in the endocardium during diabetes has been previously demonstrated using immunohistochemistry in rodents.[25] Moreover, VCAM-1 is known to be expressed in pathological conditions in the human heart.[26] During LPS-induced systemic inflammation, the widespread overexpression of VCAM-1 has been previously demonstrated by immunohistochemistry and quantitative polymerase chain reaction.[18, 27, 28] The effect of rhabdomyolysis is also supported by previous studies, including ours, showing upregulation of VCAM- 1 and recruitment of monocytes in the days following glycerol 
injection.[13] Our findings on diabetes induced VCAM-1 upregulation in the kidney are also supported by numerous studies in both mice[29] and humans[30]. Altogether, these data supports the reliability of molecular imaging to measure VCAM-1 expression in a wide range of physiological and pathophysiological conditions. It should be however acknowledged that the patterns of VCAM-1 expression should be further confirmed by other methods and in other experimental models (especially in the heart, where immunofluorescence data are more difficult to analyze due to autofluorescence) and that the pathophysiological role of endothelial activation in the studied conditions still remains to be determined.

The major obstacle to the direct application in patients of current generation MPIO- $\alpha$ VCAM-1 concerns their safety i.e. their poor biodegradability and poor stability due to spontaneous aggregation in their actual formulation. The liver and spleen mediate MPIO elimination:[1, 31] minimal retention in the kidneys or lungs has been previously observed in animal models. Several works are in progress to improve their biocompatibility and biodegradable microparticles are now available[32]. Besides toxicity issues, translation of MPIO-based molecular MRI from bench to bedside will require the demonstration of the feasibility to detect MPIO using magnet at clinical field strength and using standard coils. Indeed, the sensitivity of MPIO detection depends both on the field strength (higher field strength would produce larger susceptibility effect of the MPIOs) and on the spatial resolution of the MRI acquisition. This last parameter would be crucial to optimize for clinical translation since the resolution achieved in preclinical study is below $80 \mu \mathrm{m}$ (isotropic) and allows single MPIO imaging whereas the highest resolution of 3D $\mathrm{T} 2{ }^{*}$-weighted images on clinical magnet is around 500 $\mu \mathrm{m}$. This lower resolution, because of partial volume effects, will lower the sensitivity of the MRI acquisition to detect MPIOs.

The present method is also not appropriate for imaging the liver (due to unspecific uptake of circulating MPIO, Fig. S6). Further studies are needed to characterize MPIO- $\alpha$ VCAM-1 binding on the activated endothelium from large arteries. This could be especially important in a clinical context to detect atherosclerosis-prone segments of arteries.[33-35] Another limitation from the use of iron-oxide based contrast agents would occur in case of the presence of microhemorrhage-induced dark spots on T2*-weighted imaging, which can be misinterpreted as MPIO- $\alpha$ VCAM-1-induced signal void. The use of a short preparation period of normobaric hyperoxia may improve the specificity of the method in this context[36, 37]. Besides, the lack of iodine is an ad- vantage of MPIO in the contexts of AKI and chronic kidney disease[38]. Another limitation may arise from the influence of the shear stress on the efficiency of MPIO binding to the endothelium. Indeed, MPIO binding is more efficient at lower shear rates[15], suggesting that VCAM-1 in veins would lead to more efficient MPIO- $\alpha$ VCAM-1 binding than VCAM-1 in arteries. Therefore, the volume of MPIO- $\alpha$ VCAM-1 induced signal voids may be influenced not only by the level of VCAM-1 expression, but also by the pattern of VCAM-1 expression in the imaged organ.

On the other hand, the advantages of the present MRI method compared to currently available preclinical and clinical approaches are numerous: MPIO- $\alpha$ VCAM-1 enhanced MRI is a fast, radiation-free and non-invasive method, which allows repeated endothelial activation monitoring. Our results provide evidence that activated endothelial cells detected by conventional molecular imaging methods in acute and chronic disorders represent only the tip of the iceberg. Notably, the administered iron (1.0 $\mathrm{mg} / \mathrm{kg})$ and antibody $(150 \mu \mathrm{g} / \mathrm{kg})$ doses, the delay between injection and imaging ( $<10$ minutes) and the acquisition time (between 4 seconds for low resolution and 12 minutes for high-resolution) remain within acceptable values for clinical translation. These characteristics constitute significant improvements over previously described approaches of molecular MRI.[1, 22, 39, 40]

In conclusion, we demonstrate the feasibility of multi-organ mapping of endothelial activation in various experimental contexts using contrast-enhanced MRI. This methodology allows taking a snapshot of the endothelial activation status in multiple organs at the same time. This proof of concept in mice may serve as a basis for further development of this approach for human use.

\section{Abbreviations}

AKI: Acute Kidney Injury; BUN: Blood Urea Nitrogen; ICAM-1: Intercellular adhesion molecule-1; LPS: Lipopolysaccharide; MPIO: Micron-sized Particles of Iron Oxide; MRI: Magnetic Resonance Imaging; STZ: Streptozotocin; USPIO: Ultrasmall Superparamagnetic Particles of Iron Oxide; VCAM-1: Vascular cell adhesion molecule-1.

\section{Supplementary Material}

Figures S1-S8. http://www.thno.org/v05p1187s1.pdf

\section{Acknowledgement}

This work was supported by grants from the Institut National de la Santé Et de la Recherche Médicale (INSERM). 


\section{Competing Interests}

The authors have declared that no competing interest exists.

\section{References}

1. McAteer MA, Sibson NR, von Zur Muhlen C, Schneider JE, Lowe AS, Warrick $\mathrm{N}$, et al. In vivo magnetic resonance imaging of acute brain inflammation using microparticles of iron oxide. Nature medicine. 2007; 13: 1253-8.

2. Hasegawa Y, Saito T, Ogihara T, Ishigaki Y, Yamada T, Imai J, et al. Blockade of the nuclear factor-kappaB pathway in the endothelium prevents insulin resistance and prolongs life spans. Circulation. 2012; 125: 1122-33.

3. Kim JK. Endothelial nuclear factor kappaB in obesity and aging: is endothelial nuclear factor kappaB a master regulator of inflammation and insulin resistance? Circulation. 2012; 125: 1081-3.

4. Jefferson A, Ruparelia N, Choudhury RP. Exogenous microparticles of iron oxide bind to activated endothelial cells but, unlike monocytes, do not trigger an endothelial response. Theranostics. 2013; 3: 428-36.

5. Leuschner F, Nahrendorf M. Molecular imaging of coronary atherosclerosis and myocardial infarction: considerations for the bench and perspectives for the clinic. Circulation research. 2011; 108: 593-606.

6. Quillard T, Libby P. Molecular imaging of atherosclerosis for improving diagnostic and therapeutic development. Circulation research. 2012; 111: 231-44.

7. Gauberti M, Montagne A, Quenault A, Vivien D. Molecular magnetic resonance imaging of brain-immune interactions. Frontiers in cellular neuroscience. 2014; 8: 389.

8. McAteer MA, Choudhury RP. Targeted molecular imaging of vascular inflammation in cardiovascular disease using nano- and micro-sized agents. Vascul Pharmacol. 2013; 58: 31-8.

9. Mahmoudi M, Sheibani S, Milani AS, Rezaee F, Gauberti M, Dinarvand R, et al. Crucial role of the protein corona for the specific targeting of nanoparticles. Nanomedicine (London, England). 2015; 10: 215-26.

10. de Haas HJ, Arbustini E, Fuster V, Kramer CM, Narula J. Molecular imaging of the cardiac extracellular matrix. Circulation research. 2014; 114: 903-15.

11. Gauberti M, Montagne A, Marcos-Contreras OA, Le Behot A, Maubert E, Vivien D. Ultra-sensitive molecular MRI of vascular cell adhesion molecule-1 reveals a dynamic inflammatory penumbra after strokes. Stroke. 2013; 44: 1988-96

12. Homsi E, Janino P, de Faria JB. Role of caspases on cell death, inflammation, and cell cycle in glycerol-induced acute renal failure. Kidney Int. 2006; 69: 1385-92.

13. Belliere J, Casemayou A, Ducasse L, Zakaroff-Girard A, Martins F, Iacovoni JS, et al. Specific Macrophage Subtypes Influence the Progression of Rhabdomyolysis-Induced Kidney Injury. J Am Soc Nephrol. 2014.

14. Schanstra JP, Bachvarova M, Neau E, Bascands JL, Bachvarov D. Gene expression profiling in the remnant kidney model of wild type and kinin B1 and B2 receptor knockout mice. Kidney Int. 2007; 72: 442-54.

15. Jefferson A, Wijesurendra RS, McAteer MA, Digby JE, Douglas G, Bannister T, et al. Molecular imaging with optical coherence tomography using ligand-conjugated microparticles that detect activated endothelial cells: rational design through target quantification. Atherosclerosis. 2011; 219: 579-87.

16. Gauberti M, Martinez de Lizarrondo S, Orset C, Vivien D. Lack of secondary microthrombosis after thrombin-induced stroke in mice and non-human primates. Journal of thrombosis and haemostasis : JTH. 2014; 12: 409-14

17. Gakuba C, Gauberti M, Mazighi M, Defer G, Hanouz JL, Vivien D. Preclinical evidence toward the use of ketamine for recombinant tissue-type plasminogen activator-mediated thrombolysis under anesthesia or sedation. Stroke. 2011; 42: $2947-9$

18. Montagne A, Gauberti M, Macrez R, Jullienne A, Briens A, Raynaud JS, et al. Ultra-sensitive molecular MRI of cerebrovascular cell activation enables early detection of chronic central nervous system disorders. NeuroImage. 2012; 63: 760-70

19. Le Behot A, Gauberti M, Martinez De Lizarrondo S, Montagne A, Lemarchand E, Repesse Y, et al. GpIbalpha-VWF blockade restores vessel patency by dissolving platelet aggregates formed under very high shear rate in mice. Blood. 2014; 123: 3354-63.

20. Melemenidis S, Jefferson A, Ruparelia N, Akhtar AM, Xie J, Allen D, et al Molecular magnetic resonance imaging of angiogenesis in vivo using polyvalent cyclic RGD-iron oxide microparticle conjugates. Theranostics. 2015; 5: 515-29.

21. Franceschi C, Bonafe M, Valensin S, Olivieri F, De Luca M, Ottaviani E, et al. Inflamm-aging. An evolutionary perspective on immunosenescence. Annals of the New York Academy of Sciences. 2000; 908: 244-54.

22. Weissleder R, Nahrendorf M, Pittet MJ. Imaging macrophages with nanoparticles. Nature materials. 2014; 13: 125-38.

23. Majmudar MD, Yoo J, Keliher EJ, Truelove JJ, Iwamoto Y, Sena B, et al. Polymeric nanoparticle PET/MR imaging allows macrophage detection in atherosclerotic plaques. Circulation research. 2013; 112: 755-61.

24. Shlipak MG, Fried LF, Crump C, Bleyer AJ, Manolio TA, Tracy RP, et al. Elevations of inflammatory and procoagulant biomarkers in elderly persons with renal insufficiency. Circulation. 2003; 107: 87-92.
25. Popov D, Sima A, Stern D, Simionescu M. The pathomorphological alterations of endocardial endothelium in experimental diabetes and diabetes associated with hyperlipidemia. Acta diabetologica. 1996; 33: 41-7.

26. Yamashita T, Sekiguchi A, Iwasaki YK, Date T, Sagara K, Tanabe H, et al Recruitment of immune cells across atrial endocardium in human atrial fibrillation. Circulation journal : official journal of the Japanese Circulation Society. 2010; 74: 262-70.

27. Kurniati NF, van Meurs M, Vom Hagen F, Jongman RM, Moser J, Zwiers PJ, et al. Pleiotropic effects of angiopoietin-2 deficiency do not protect mice against endotoxin-induced acute kidney injury. Nephrology, dialysis, transplantation official publication of the European Dialysis and Transplant Association European Renal Association. 2013; 28: 567-75.

28. Raeburn CD, Calkins CM, Zimmerman MA, Song Y, Ao L, Banerjee A, et al. ICAM-1 and VCAM-1 mediate endotoxemic myocardial dysfunction independent of neutrophil accumulation. American journal of physiology Regulatory, integrative and comparative physiology. 2002; 283: R477-86.

29. Omote K, Gohda T, Murakoshi M, Sasaki Y, Kazuno S, Fujimura T, et al. Role of the TNF pathway in the progression of diabetic nephropathy in KK-A(y) mice. American journal of physiology Renal physiology. 2014; 306: F1335-47.

30. Liu JJ, Yeoh LY, Sum CF, Tavintharan S, Ng XW, Liu S, et al. Vascular cell adhesion molecule-1, but not intercellular adhesion molecule-1, is associated with diabetic kidney disease in Asians with type 2 diabetes. Journal of diabetes and its complications. 2015; 29: 707-12.

31. Akhtar AM, Schneider JE, Chapman SJ, Jefferson A, Digby JE, Mankia K, et al. In vivo quantification of VCAM-1 expression in renal ischemia reperfusion injury using non-invasive magnetic resonance molecular imaging. PloS one. 2010; 5: e12800.

32. Jefferson A, Wijesurendra RS, McAteer MA, Choudhury RP. Development and application of endothelium-targeted microparticles for molecular magnetic resonance imaging. Wiley Interdiscip Rev Nanomed Nanobiotechnol. 2012; 4: 247-56

33. Mulder WJ, Jaffer FA, Fayad ZA, Nahrendorf M. Imaging and nanomedicine in inflammatory atherosclerosis. Science translational medicine. 2014; 6: 239sr1.

34. Chakravarty R, Goel S, Cai W. Nanobody: the "magic bullet" for molecular imaging? Theranostics. 2014; 4: 386-98.

35. Orbay $\mathrm{H}$, Hong $\mathrm{H}$, Zhang $\mathrm{Y}$, Cai W. Positron emission tomography imaging of atherosclerosis. Theranostics. 2013; 3: 894-902.

36. Gaberel T, Gakuba C, Hebert M, Montagne A, Agin V, Rubio M, et al. Intracerebral hematomas disappear on $\mathrm{T}^{*}$-weighted images during normobaric oxygen therapy. Stroke. 2013; 44: 3482-9.

37. Gaberel T, Gakuba C, Goulay R, Martinez De Lizarrondo S, Hanouz JL, Emery E, et al. Impaired glymphatic perfusion after strokes revealed by contrast-enhanced MRI: a new target for fibrinolysis? Stroke. 2014; 45: 3092-6.

38. Zhang YD, Wang J, Zhang J, Wang X, Jiang X. Effect of iodinated contrast media on renal function evaluated with dynamic three-dimensional MR renography. Radiology. 2014; 270: 409-15.

39. Thurman JM, Serkova NJ. Nanosized contrast agents to noninvasively detect kidney inflammation by magnetic resonance imaging. Adv Chronic Kidney Dis. 2013; 20: 488-99.

40. Broisat A, Hernot S, Toczek J, De Vos J, Riou LM, Martin S, et al. Nanobodies targeting mouse/human VCAM1 for the nuclear imaging of atherosclerotic lesions. Circulation research. 2012; 110: 927-37. 